\title{
Design Principles for Long-Range Energy Transfer at Room Temperature
}

\author{
Andrea Mattioni, ${ }^{\ddagger}$ Felipe Caycedo-Soler, ${ }^{*}$ Susana F. Huelga, and Martin B. Plenio ${ }^{\dagger}$ \\ Institut für Theoretische Physik and Center for Integrated Quantum Science and Technology IQST, \\ Albert-Einstein-Allee 11, Universität Ulm, 89069 Ulm, Germany
}

(Received 4 June 2019; revised 15 July 2021; accepted 19 July 2021; published 6 October 2021)

\begin{abstract}
Under physiological conditions, ballistic long-range transfer of electronic excitations in molecular aggregates is generally expected to be suppressed by noise and dissipative processes. Hence, quantum phenomena are not considered to be relevant for the design of efficient and controllable energy transfer over significant length scales and timescales. Contrary to this conventional wisdom, here we show that the robust quantum properties of small configurations of repeating clusters of molecules can be used to tune energy-transfer mechanisms that take place on much larger scales. With the support of an exactly solvable model, we demonstrate that coherent exciton delocalization and dark states within unit cells can be used to harness dissipative phenomena of varying nature (thermalization, fluorescence, nonradiative decay, and weak intersite correlations) to support classical propagation over macroscopic distances. In particular, we argue that coherent delocalization of electronic excitations over just a few pigments can drastically alter the relevant dissipation pathways that influence the energy-transfer mechanism and thus serve as a molecular control tool for large-scale properties of molecular materials. Building on these principles, we use extensive numerical simulations to demonstrate that they can explain currently not-understood measurements of micron-scale exciton diffusion in nanofabricated arrays of bacterial photosynthetic complexes. Based on these results, we provide quantum design guidelines at the molecular scale to optimize both energy-transfer speed and range over macroscopic distances in artificial light-harvesting architectures.
\end{abstract}

DOI: 10.1103/PhysRevX.11.041003

\section{INTRODUCTION}

Over the last decades, research into the role of coherent excitonic delocalization in the dynamics in photosynthetic membranes has shown that strong coherent coupling in subunits of tightly coupled pigments can result in shortranged excitonic delocalization in the steady state [1-6]. Delocalization within these domains, typically restricted to individual proteins termed antenna complexes, is essential for modeling transient and steady-state optical spectra of the full light-harvesting ensemble. Additionally, excitonic delocalization within antenna complexes is a crucial ingredient for a modular description of dynamics over

\footnotetext{
*Deceased.

† martin.plenio@uni-ulm.de

*Present address: Department of Chemistry, School of Natural Sciences, The University of Manchester, Oxford Road, Manchester, M13 9PL, United Kingdom.

Published by the American Physical Society under the terms of the Creative Commons Attribution 4.0 International license. Further distribution of this work must maintain attribution to the author(s) and the published article's title, journal citation, and DOI.
}

Subject Areas: Chemical Physics, Condensed Matter Physics, Quantum Physics

longer distances and timescales [7-9], which, as observed experimentally [10-13], can be interpreted as a series of incoherent transfer steps, described by simple rate processes. These energy-transfer rates depend on the properties of the states involved and thereby rely heavily on the steady-state excitonic delocalization within antenna complexes, as has been shown by numerically exact calculations $[4,5,14]$.

The modular architecture found in biological lightharvesting membranes, whereby antenna complexes containing a few pigments self-aggregate into larger structures, offers the potential for artificial solar energy conversion and molecular electronics based on such a modular design [15-18]. This potential is made possible by the high degree of experimental control that is available today for the integration of synthetic and biological structures and for the directed assembly of photosynthetic antenna complexes isolated from living organisms [19-24] or of supramolecular dye arrays [25-31]. To facilitate the realization of these devices, however, a theoretical understanding of the mechanisms involved in to-date unexplained observations of the large diffusion lengths in several of these light-harvesting architectures is needed. For instance, the observed micronscale diffusion of excitations in nanofabricated arrays of 
purple bacteria antenna complexes and phycobilisome proteins [19-21] exceeds, by more than 1 order of magnitude, the theoretical expectation for diffusion based on experimentally determined parameters under physiological conditions. On the one hand, this shows that an important enhancement of the diffusion can be achieved with hybrid technologies. On the other hand, it stresses the necessity for theory to establish and verify physical design principles by which delocalization of electronic excitations over a few pigments can enable the observed long-range energy transfer.

One viable strategy for improving the diffusion lengths of excitons is to extend their lifetime, thus allowing them to propagate across longer distances. Recent studies on the role of dark states in solar energy conversion have provided valuable insight into the potential advantages of protecting excitations from losses due to fluorescence in order to promote charge separation [32-36]. These models, though, do not consider the microscopic origin of the interactions, thereby omitting the conditions that enable or inhibit the active participation of dark states in the dynamics. Because dark states cannot be excited directly by light and typically do not couple efficiently to the propagating bright states, their participation in the exciton propagation is often overlooked and requires a careful reconsideration of the typical models used to describe resonant energy transfer $[37,38]$. A further challenge is presented by the need for accounting for nonradiative decay due to the interaction between electronic excitations and vibrational motion of pigment molecules, which is responsible for the usually low fluorescence yield of light-harvesting complexes $[39,40]$. In fact, the competition between radiative and nonradiative decay channels can drastically modify the dissipation landscape, leading to scenarios in which optically dark states have a much shorter lifetime than bright states. Moreover, the dynamics of excitons can be influenced by the presence of correlations between the local vibrational environments of each pigment. Most research on the topic has been focused on clarifying the extent to which these correlations influence the ultrafast spectroscopy of excitons, often leading to contrasting predictions [41]. However, more relevant for our case, weak intersite correlations can be expected to play a role on much longer timescales, too, and thus influence nonradiative decay.

In the present work, we provide a theoretical model that identifies the desirable features of spectral structures and exciton delocalization within unit cells (subunits of tightly coupled pigments) that support efficient energy transfer. This model builds on the formation and participation of dark states in the dynamics in order to achieve long-range diffusion across arrays of these subunits. We show how this is possible by a combination of excitonic delocalization within unit cells and close proximity between unit cells. Identifying each LH2 complex as a unit cell of the transfer chain, we show that a model that can explain the long-range energy propagation reported in LH2 arrays does not need to resort to previously hypothesized [42-44], long-range quantum coherence involving several LH2 complexes [39,45-48]. Indeed, we demonstrate that a theoretical description that is consistent with available experimental data concerning structure and optical response of the LH2 antenna [39,45-47,49] can be developed, which reproduces the experimentally observed exciton diffusion length [20]. This theoretical description therefore presents desirable features for transport across photosynthetic membranes. The low-energy part of the excitonic spectrum of a unit cell comprises states that are protected against dissipation, while high-energy excitons offer fast pathways for energy transfer to neighboring units by virtue of their delocalization. A combination of these two features allows for robust long-range energy transfer.

The remainder of this work is organized as follows. In Sec. II A, we present the exactly solvable model, which contains the necessary features to discuss long-range exciton propagation across a modular array of excitonic unit cells. Here, we discuss several energy-transfer regimes and their relation to the underlying excitonic properties. In Sec. II B, we introduce the bacterial antenna complex LH2 and provide a thorough characterization of their excitons relevant to energy transfer. In Sec. II C, we present the results of our simulations of a linear array of LH2 complexes, and we discuss how the mechanisms presented in Sec. II A apply to a real-world application, backed by existing experimental findings. Section III summarizes the main results of this work and sets a broader context in which these can provide useful guidelines for energytransfer design in molecular materials.

\section{MODEL, RESULTS, AND MAJOR INSIGHTS}

\section{A. Minimal model of a light-harvesting array}

The beneficial role provided by exciton delocalization and the formation of states protected against dissipation in excitonic energy transfer can be readily understood in a model system made up of dimeric unit cells [Fig. 1(a)]. Let us consider a homogeneous linear array of unit cells, each of them described by a Hamiltonian

$$
\hat{H}^{(n)}=\sum_{i} \varepsilon_{i}\left|i_{n}\right\rangle\left\langle i_{n}\left|+\sum_{i \neq j} J_{i j}\right| i_{n}\right\rangle\left\langle j_{n}\right|,
$$

where the state $\left|i_{n}\right\rangle$ describes an electronic excitation of the $i$ th pigment belonging to the $n$th unit cell. Energy transfer across the full array is made possible by the interaction between pigments of different unit cells, described by $\hat{V}^{(n, n+1)}=\sum_{i, j} V_{i j}\left|i_{n}\right\rangle\left\langle j_{n+1}\right|+$ H.c., where $V_{i j}$ are interdimer dipole-dipole couplings.

If the single-pigment dephasing rate $\gamma$, the intradimer and interdimer couplings $J_{12}$, and the interdimer dipole-dipole couplings $V_{i j}$ follow 


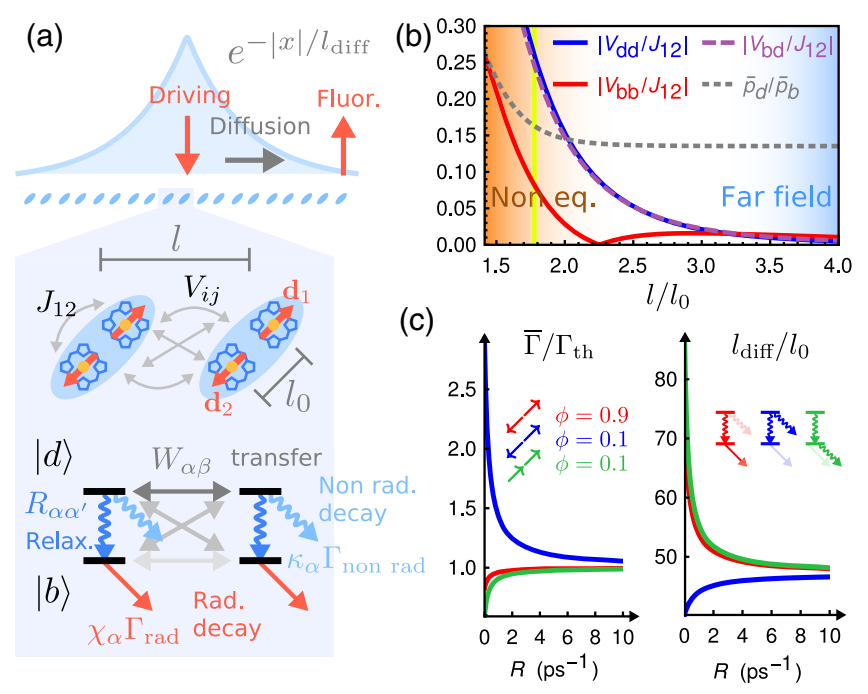

FIG. 1. Exciton propagation across a chain of dimerized units. (a) Linear chain of dimerized unit cells separated by a distance $l$ consisting of interacting transition dipole moments, and a sketch of the geometry and level diagram of two neighboring unit cells. Energy transfer between subunits is considered to be incoherent. (b) Distance dependence of the couplings between unit-cell excitons. For large distances (far field, $l / l_{0} \gtrsim 3.2$ ), the coupling between bright states $V_{b b}$ dominates (red line). At short distances, dark states are mainly involved in the energy transfer, which proceeds through the couplings $V_{d d}$ and $V_{b d}$ (blue and purple lines). Because of these couplings, the stationary populations $\bar{p}_{b}$ and $\bar{p}_{d}$ deviate from those in the thermal equilibrium regime that characterizes the far-field limit (gray dotted line). (c) Effective decay rate $\bar{\Gamma}$ (left) and diffusion length $l_{\text {diff }}$ (right) as a function of the local exciton-phonon coupling, quantified by $R=\sqrt{R_{b d} R_{d b}}$. The interdimer distance is fixed to $l / l_{0}=1.78$, corresponding to the yellow line in (a). For low fluorescence yield and antiparallel dipoles (blue line), the coupling to local phonons is beneficial for long-range energy transfer as a consequence of a reduced decay rate. A larger fluorescence yield (red line) or a change in exciton symmetry (green line) leads to an unfavorable scaling with respect to $R$ : Coupling to local phonons hinders long-range energy transfer. The model parameters take the values $\gamma=(30 \mathrm{fs})^{-1}=177 \mathrm{~cm}^{-1} / \hbar, l_{0}=1 \mathrm{~nm}, d=5 \mathrm{D}, \Gamma_{\text {th }}=$ $(1 \mathrm{~ns})^{-1}, e^{-l_{0} / r_{\mathrm{c}}}=0.9$, and $k_{\mathrm{B}} T=J_{12}=252 \mathrm{~cm}^{-1}$.

$$
V_{i j}<\hbar \gamma<J_{12}
$$

then delocalized intradimer excitons defined by $\hat{H}^{(n)}\left|\alpha_{n}\right\rangle=$ $E_{\alpha}\left|\alpha_{n}\right\rangle$ form. These may be used to describe the (incoherent) energy transfer between adjacent dimers [8]. We refer to "incoherent" or "classical" transfer interchangeably, meaning that interunit cell coherences of the density operator of the full chain are negligible $\left(\left\langle i_{n}|\rho| j_{m}\right\rangle \approx 0\right.$ for $n \neq m$ ), which then results in classical diffusion across subunits exhibiting local coherent dynamics. The hierarchy of interactions in Eq. (2) is commonly fulfilled in photosynthetic membranes and nano-engineered arrays, where pigments aggregate in antenna complexes within subnanometer distances, while the intercomplex distances can span several nanometers, as we will discuss later in detail. For the configuration of antiparallel transition dipoles (i.e., $\mathbf{d}_{1}=-\mathbf{d}_{2}=d \mathbf{e}_{1}$ ) [indicated by red arrows in Fig. 1(a)], the $n$th dimeric unit supports a dark (bright) exciton $\left|d_{n}\right\rangle\left(\left|b_{n}\right\rangle\right)$ given by the symmetric (antisymmetric) coherent superposition $\left|\alpha_{n}\right\rangle$ of single-pigment states, namely, $\left.\left|\alpha_{n}\right\rangle=||_{b_{n}}^{d_{n}}\right\rangle=\left(\left|1_{n}\right\rangle \pm\left|2_{n}\right\rangle\right) / \sqrt{2}$ when $\varepsilon_{1}=\varepsilon_{2}$.

Within this framework, a quantum master equation description of the full chain Hamiltonian in the presence of dephasing and relaxation mechanisms [50] can be replaced by the classical rate equations

$$
\begin{aligned}
\partial_{t} p_{\alpha}^{n}= & -\Gamma_{\alpha} p_{\alpha}^{n}-\sum_{\beta, m=n \pm 1} W_{\beta \alpha} p_{\alpha}^{n}+\sum_{\beta, m=n \pm 1} W_{\alpha \beta} p_{\beta}^{m} \\
& -\sum_{\alpha^{\prime}(\neq \alpha)} R_{\alpha^{\prime} \alpha} p_{\alpha}^{n}+\sum_{\alpha^{\prime}(\neq \alpha)} R_{\alpha \alpha^{\prime}} p_{\alpha^{\prime}}^{n}+I_{\alpha}^{n},
\end{aligned}
$$

for the population $p_{\alpha}^{n}$ of the $\alpha$ th exciton on the $n$th dimeric unit cell. Here, pairs of subindices $\alpha \beta\left(\alpha \alpha^{\prime}\right)$ label excitons on different (the same) unit cells. The rates that describe the transfer of excitations between unit cells, $W_{\alpha \beta}$, their overall decay rate $\Gamma_{\alpha}$, and their thermalization rate $R_{\alpha \alpha^{\prime}}$ depend on the characteristics of the quantum states and their environments within these cells. Injection of excitations into the array occurs with rates $I_{\alpha}^{n}$ on site $n$. The rates $W_{\alpha \beta}$ can be obtained from the overlap between homogeneous lineshape functions and depend on the coupling matrix elements $\quad V_{\alpha \beta}=\left\langle\alpha_{n}\left|\hat{V}^{(n, n+1)}\right| \beta_{n+1}\right\rangle \quad$ between unit-cell eigenvectors and the relative dephasing rate $\gamma_{\alpha \beta}$ between these states via

$$
W_{\alpha \beta}= \begin{cases}\frac{2\left|V_{\alpha \beta}\right|^{2}}{\hbar^{2}} \frac{1}{\gamma_{\alpha \beta}} & \text { for } \alpha=\beta \\ \frac{2\left|V_{\alpha \beta}\right|^{2}}{\hbar^{2}} \frac{\gamma_{\alpha \beta}}{\gamma_{\alpha \beta}^{2}+4 J_{12}^{2} / \hbar^{2}} & \text { otherwise }\end{cases}
$$

as explained in more detail in the Appendix D. The dephasing rate between nonoverlapping excitons $\gamma_{\alpha \beta}=$ $\gamma_{\alpha}+\gamma_{\beta}$ is the sum of the linewidths $\gamma_{\alpha}=\sum_{\alpha^{\prime}} R_{\alpha^{\prime} \alpha} / 2$, which are typically dominated by pure dephasing $R_{\alpha \alpha}$ over the intraunit-cell thermalization rates $R_{\alpha^{\prime} \neq \alpha}$. The thermalization rate $R_{\alpha \neq \alpha^{\prime}} \propto \mathcal{J}\left(\left|E_{\alpha}-E_{\alpha^{\prime}}\right|\right)\left|n\left(E_{\alpha}-E_{\alpha^{\prime}}\right)\right|$ is proportional to the phonon spectral density $\mathcal{J}(\hbar \omega)$ and to the thermal boson occupation number $n(\hbar \omega)$ across the excitonic manifold, which leads to a Boltzmann distribution of the excitons when injection, loss, and interunit-cell transfer are much slower than thermalization. Excitonic pure dephasing $R_{\alpha \alpha} / 2=\sum_{i}\left|\left\langle i_{n} \mid \alpha_{n}\right\rangle\right|^{4} \gamma \equiv \mathcal{P}_{\alpha}^{-1} \gamma$ is slower than the pigment's pure dephasing $\gamma$ by a factor given by the inverse participation ratio $\mathcal{P}_{\alpha}^{-1}$ [51]. Consequently, excitonic delocalization $\left(\mathcal{P}_{\alpha}>1\right)$ results in slower dephasing rates for unit-cell excitons in comparison to individual pigments, which, based on Eq. (4), implies an enhancement 
of the transfer rates between excitons in neighboring units with increasing unit-cell delocalization. For this dimeric system where $\mathcal{P}_{\alpha}=2$, delocalization over two pigments of states $\left|b_{n}\right\rangle$ and $\left|d_{n}\right\rangle$ results in a twofold speedup on the interdimer transfer $W_{d d}$ and $W_{b b}$, as compared to unit cells where strong dephasing $\gamma$ or mild coupling $J_{12}$ prevents the formation of delocalized excitons $\left|\alpha_{n}\right\rangle$. Excitonic delocalization within unit cells also redistributes the optical transition dipole strength of individual pigments, resulting in a fluorescence rate of excitons $\chi_{\alpha} \Gamma_{\text {rad }}$, where $\Gamma_{\text {rad }}$ is the single-pigment fluorescence rate and $\chi_{\alpha}=$ $\sum_{i j}\left\langle\alpha_{n} \mid i_{n}\right\rangle \chi_{i j}\left\langle j_{n} \mid \alpha_{n}\right\rangle$ characterizes the optical brightness of an exciton. The brightness quantifies the number of sites participating in the fluorescence from exciton $\alpha$ [52] and is determined by $\chi_{i j}=\left(\mathbf{e}_{i} \cdot \mathbf{e}_{j}\right) j_{0}\left(2 \pi r_{i j} / \lambda\right)-\frac{1}{2}\left[\mathbf{e}_{i}\right.$. $\left.\mathbf{e}_{j}-3\left(\mathbf{e}_{i} \cdot \mathbf{n}_{i j}\right)\left(\mathbf{e}_{j} \cdot \mathbf{n}_{i j}\right)\right] j_{2}\left(2 \pi r_{i j} / \lambda\right)$, where $j_{\nu}$ are spherical Bessel functions of the first kind, $\lambda$ is the wavelength associated with the pigment's $Q_{y}$ transition, and $\mathbf{r}_{i j}=$ $r_{i j} \mathbf{n}_{i j}$ is the relative position of pigments $i$ and $j$. In the limit $r_{i j} \ll \lambda$, the brightness reduces to the usual measure of superradiance $\left|\mathbf{D}_{\alpha}\right|^{2} / d^{2}$, i.e., the relative dipole strength, where $\mathbf{D}_{\alpha}=\sum_{i}\langle i \mid \alpha\rangle \mathbf{d}_{i}$, leading to a superradiant $|b\rangle$ $\left(\chi_{b} \approx 2\right)$ and a subradiant $|d\rangle\left(\chi_{d} \approx 0\right)$ exciton for the dimeric unit cells of Fig. 1(a).

Two ideas that will play a major role later are worth stressing at this point. First, we note that, while dark states do not couple significantly to electromagnetic fields, they still play a central role in the energy-transfer dynamics across different unit cells. Intuitively, one expects that, if the distance between unit cells is much larger than their internal size $\left(l \gg l_{0}\right)$, two neighboring dimers couple via their global dipoles $\mathbf{D}_{\alpha}$. However, if the dimers are placed sufficiently close such that their distance becomes comparable to their spatial extent $\left(l \gtrsim l_{0}\right)$, the dipole approximation for their mutual interaction breaks down, and states with vanishing dipole strength can start to interact via their higher moments, thus gaining some coupling strength $V_{d d}$. This interaction can even exceed the one of bright states $V_{b b}$ in certain configurations, as shown in Fig. 1(b), where the coupling strengths $V_{\alpha \beta}$ are plotted against the interdimer distance, for the arrangement shown in Fig. 1(a). For distances $l \lesssim 3.2 l_{0}$, dark states couple more strongly than bright states. As a result, energy transfer through the dark manifold can be achieved much faster than through its bright counterpart. Second, we note that typical lightharvesting complexes show a rather low quantum yield of fluorescence $\phi$; i.e., most of the optically generated excitations are lost through nonradiative decay channels. Thus, dark states, although protected against fluorescence, could easily be more dissipative than bright states, as we show below. As discussed in Appendixes B and C, nonradiative decay rates are influenced by the presence of correlations in the vibrational environments of single pigments. When these correlations are taken into account, the nonradiative decay rate is distributed to different excitons, analogously to what happens to the radiative rates (i.e., superradiance). In fact, static correlations between local vibrational environments can be interpreted as arising from the presence of delocalized vibrations coupling to different sites (see Appendix C for a detailed explanation). Under these circumstances, the excitonic decay rates gain a prefactor $\kappa_{\alpha}$, which depends on the phase with which each site contributes to the excitonic wave function. Depending on the specific exciton, localized excitations can interfere constructively (destructively) to yield nonradiative rates that can be larger (smaller) than their single-pigment counterpart. In our case, the nonradiative decay rate of the symmetric (antisymmetric) state $\left|d_{n}\right\rangle\left(\left|b_{n}\right\rangle\right)$ is enhanced (reduced) by a factor $\kappa_{d(b)}=1 \pm e^{-l_{0} / r_{\mathrm{c}}}$ with respect to its single-pigment value $\Gamma_{\text {nonrad }}$, where $r_{\mathrm{c}}$ is the correlation length of the pigment's vibrational environment. Thus, the decay rate from exciton $\left|\alpha_{n}\right\rangle$ is given by

$$
\Gamma_{\alpha}=\chi_{\alpha} \Gamma_{\text {rad }}+\kappa_{\alpha} \Gamma_{\text {nonrad }} .
$$

Note that, for large correlation lengths, the nonradiative decay from the bright state can be significantly reduced compared to the one from the dark state, i.e., $\kappa_{b} \ll \kappa_{d}$. Since both radiative and nonradiative decays in lightharvesting complexes are typically much slower than exciton thermalization, the decay of photoexcitations in a single light-harvesting unit occurs from a thermalized exciton distribution with the average rate

$$
\Gamma_{\mathrm{th}}=\sum_{\alpha} p_{\alpha}^{\mathrm{th}} \Gamma_{\alpha}
$$

where $p_{\alpha}^{\text {th }} \propto e^{-E_{\alpha} / k_{\mathrm{B}} T}$. This average decay rate is an experimentally accessible quantity, as is the quantum yield of fluorescence,

$$
\phi=\frac{\sum_{\alpha} \chi_{\alpha} p_{\alpha}^{\mathrm{th}} \Gamma_{\mathrm{rad}}}{\Gamma_{\mathrm{th}}}
$$

The latter only determines the overall relative contribution of radiative and nonradiative decays, whereas the factors $\chi_{\alpha}$ and $\kappa_{\alpha}$ determine how these rates are distributed across the excitonic manifold.

So far, we have discussed how delocalization, transition dipole geometry and environmental correlations determine the dissipation properties of the excitonic states within unit cells, radiative and nonradiative alike, and how the finite size of the unit cell in densely packed arrays opens up the possibility to engage dark states into the propagation dynamics. We now have all the necessary ingredients to determine how all these properties influence the diffusion length of excitons across the light-harvesting array. As we are interested in diffusion over macroscopic distances, it is useful to describe the position of a dimeric unit cell in terms 
of a continuous variable $x=n l$ as $l \rightarrow 0$. Thus, bright- and dark-state populations at discrete sites $p_{\alpha}^{n}(t)$ are replaced by the respective densities $p_{\alpha}(x, t)$, and Eq. (3) takes the form of two, coupled, continuous diffusion equations, which allow for an analytical solution of the steady-state density $p_{\alpha}(x, \infty)$, as detailed in Appendix A. If we assume that the injection and decay happen on a much slower timescale than transfer and relaxation, the solutions for local driving at $x=0$ take the simple form $p_{\alpha}(x, \infty)=\left[\bar{p}_{\alpha} /\left(2 l_{\text {diff }}\right)\right] e^{-|x| / l_{\text {diff }}}$, with $\bar{p}_{b}+\bar{p}_{d}=1$. The diffusion length is given by $l_{\text {diff }}=l \sqrt{\bar{W} / \bar{\Gamma}}$, introducing the effective transfer and decay rates

$$
\begin{gathered}
\bar{W}=\bar{p}_{b}\left(W_{b b}+W_{d b}\right)+\bar{p}_{d}\left(W_{d d}+W_{b d}\right), \\
\bar{\Gamma}=\bar{p}_{b} \Gamma_{b}+\bar{p}_{d} \Gamma_{d},
\end{gathered}
$$

as weighted averages on the populations $\bar{p}_{\alpha}$. These populations are determined by the ratio

$$
\frac{\bar{p}_{d}}{\bar{p}_{b}}=\frac{R_{d b}+2 W_{d b}}{R_{b d}+2 W_{b d}}
$$

which only depends on the rates that provide mixing between bright and dark manifolds. In particular, we observe that the presence of symmetric interdimer bright-to-dark transfer $W_{d b}=W_{b d}$ causes a deviation of the steady-state populations from thermal equilibrium $R_{d b} / R_{b d}=e^{-\left(E_{d}-E_{b}\right) / k_{\mathrm{B}} T}$, resulting in an increase of the stationary dark-state population. Thus, the onset of this nonequilibrium state, which can be observed at short interdimer separations $l$ when the rates $W_{b d}=W_{d b}$ become comparable to $R_{b d}$ and $R_{d b}$ [Fig. 1(b), gray dotted line], leads to a modification of effective transfer and decay rates with respect to their equilibrium values.

In order to study the effect of dark states on energy transfer, we focus on the dependence of the diffusion length $l_{\text {diff }}$ on the average intradimer relaxation rate $R=\sqrt{R_{b d} R_{d b}}$, quantifying the strength of local electron-phonon coupling, at a fixed lattice spacing $l$ such that bright-to-dark transfer and relaxation take place on a similar timescale and exciton transfer through dark states is faster than through bright states [Fig. 1(b), yellow vertical line].

First, we consider the ideal situation in which the main decay channel is radiative $(\phi=0.9)$ : Most of the dissipation takes place at the low-energy bright state, which propagates more slowly than the higher-energy dark exciton. In this scenario, long-range energy transfer clearly benefits from the establishment of a nonequilibrium state with more population in the dark state. In fact, when reducing $R$, e.g., by decreasing the local exciton-phonon coupling, we are moving population from the slow, dissipative bright state to the fast, less-dissipative dark state. Furthermore, at the same time, we are reducing the effective decay rate $\bar{\Gamma}$ and increasing the effective transfer rate $\bar{W}$, resulting in a larger diffusion length $l_{\text {diff }}$ [Fig. 1(c), red line].

In a more realistic scenario, we expect nonradiative decay to play a much bigger role. Let us then set $\phi=0.1$, closer to values observed for biological light-harvesting complexes. In doing so, we also adjust the single-pigment decay rates $\Gamma_{\text {rad }}$ and $\Gamma_{\text {nonrad }}$ to ensure that the effective decay rate at thermal equilibrium $\Gamma_{\text {th }}$ from Eq. (6) remains the same as in the case just considered. Since the main decay pathway is now the nonradiative decay from the fast high-energy exciton, an increase in the relaxation rate $R$ would lead to a longer-lived excitation but also to a slower propagation. However, the former prevails: The reduction of effective decay $\bar{\Gamma}$ is large enough to ensure a longerranged propagation [Fig. 1(c), blue line]. In other words, by increasing the coupling to local phonons, we counterintuitively increase the spatial extent of energy transfer, at the price of making it slower. This energy-transfer regime can be seen as a natural extension to systems with realistic nonradiative decay channels of the dark-state protection scheme proposed in the context of quantum photocells [32-36] and recently applied to energy transfer [53]. Our generalized "dark" state protection mechanism makes use of states that are protected against nonradiative decay (and are therefore "dark") to extend the lifetime of the excitons, which can diffuse across longer distances.

Lastly, we observe that, while nonradiative decay pathways seem to fundamentally limit the transport efficiency of light-harvesting architectures, one can work around this constraint by exploiting the symmetry of the excitonic wave function. Let us consider an arrangement in which the dipoles within each dimeric unit cell are now parallel. In this situation, the intradimer coupling $J_{12}$ becomes negative, and the low-energy exciton is now the symmetric state $\left(\left|1_{n}\right\rangle+\left|2_{n}\right\rangle\right) \sqrt{2}$. While most of the optical dipole still resides in the low energy level, this state also becomes the most sensitive to nonradiative decay. Thus, we are left with a high-energy exciton, which shows little dissipation and fast transfer. As shown in Fig. 1(c) (green line), this situation is similar to the one that we considered with $\phi=0.9$. The important difference, however, is that, in this case, nonradiative decay is fully taken into account, but its effect is mitigated by exploiting excitonic delocalization within a unit cell and the ensuing redistribution of decay pathways.

\section{B. Bacterial light-harvesting units}

The simple dynamical model considered so far allows for analytical expressions that facilitate the identification of the different mechanisms on which we base our explanation of the to-date unexplained experimental observations of longrange energy transfer in nano-engineered arrays of LH2 photosynthetic complexes of purple bacteria $R b$. sphaeroides [20]. These complexes consist of a protein holding 

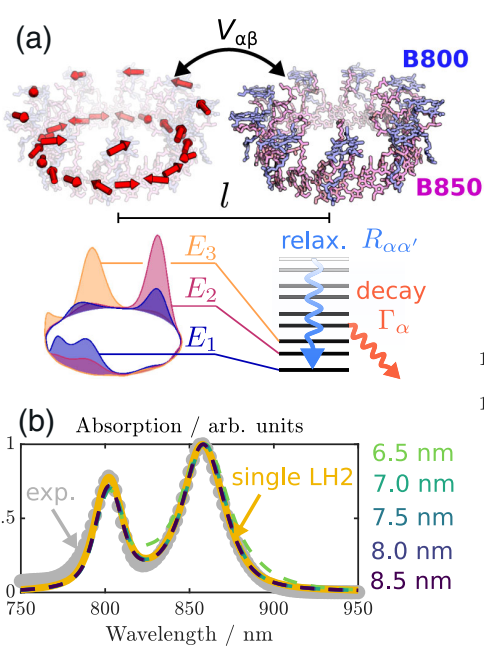

(c)
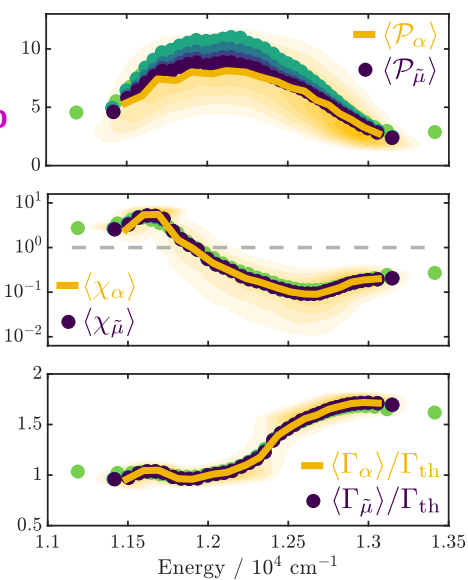

(d)
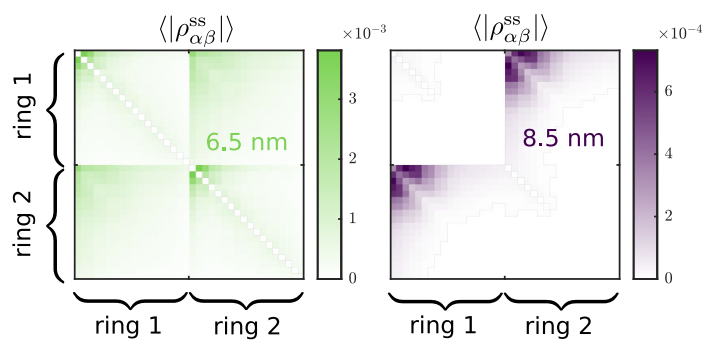

(e)
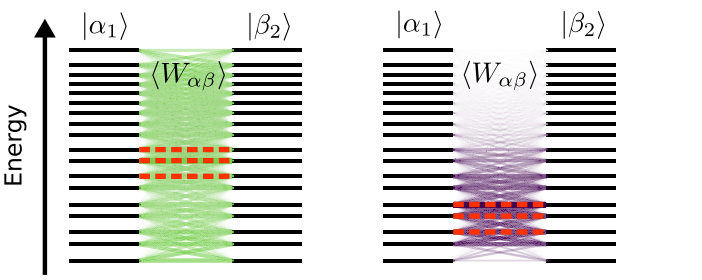

FIG. 2. Excitons in bacterial light-harvesting units. (a) Pigment arrangement in a LH2 antenna from Rps. acidophila [49]. B850 (violet) and B800 (blue) rings are composed, respectively, of 18 and 9 BChls, whose $Q_{y}$ transition dipoles are indicated by red arrows. Electronic excitations are partially delocalized and undergo relaxation within the exciton manifold; they decay to the ground state or transfer to neighboring rings. (b) Experimental absorption spectrum [55] (gray dots) and theoretical fit of the B800 and B850 bands of LH2. The inclusion of coherent inter-ring couplings in an LH2 pair (green to violet dashed lines) only leads to small deviations from the single-ring absorption (yellow line). (c) Energy distribution of the participation ratio (top), brightness (center), and decay rates (bottom) of B850 excitons, for a single ring (yellow line) and for a coherently coupled B850 pair for different inter-ring distances (green to violet dots). For a single ring, the underlying distribution is shown (yellow shading). Most delocalized states lie in the midenergy range; superradiant excitons $\left(\chi_{\alpha}>1\right.$, above the gray dashed line) occupy the low-energy end of the spectrum, whereas the states with stronger dissipation are high-energy dark states. Two-ring excitons are slightly more delocalized, but all two-ring quantities lie within the singlering distributions. (d) Steady-state density matrix of two coherently coupled B850 rings undergoing local relaxation and dephasing in the single-ring exciton basis, for $l=6.5 \mathrm{~nm}$ (green) and $8.5 \mathrm{~nm}$ (violet). Excitons are organized in ascending energy and grouped according to the ring to which they belong. Populations are set to zero to increase contrast. (e) Average energy-transfer rates $\left\langle W_{\alpha \beta}\right\rangle$ between two B850 rings for $l=6.5 \mathrm{~nm}$ (green) and $8.5 \mathrm{~nm}$ (violet). Thicker and darker lines correspond to faster transfer. The three fastest transfer pathways are highlighted (red dashed lines). For short $l$, the excitons in the mid-high-energy range are mostly involved in energy transfer. Averages are performed over $10^{4}$ realizations of static disorder.

two concentric bacteriochlorophyll (Bchl) rings [Fig. 2(a)] with the inner B850 ring consisting of 18 strongly interacting Bchl pigments, which at room temperature exhibit an excitonic delocalization across about 3-6 pigments, determined by superradiance measurements $[39,45-$ 48]. This subunit mediates the transfer between LH2 complexes under physiological conditions, whereas the B800 pigments support localized excitations that extend the absorption range of the LH2 complex and regulate oxidation [54].

We consider the full B850 Hamiltonian $\hat{H}^{(n)}$ describing the interactions among $Q_{y}$ transitions of its $18 \mathrm{BChls,}$ sketched with red arrows in Fig. 2(a), and study the delocalization properties of the single-ring excitons $|\alpha\rangle$, with $\alpha=1, \ldots, 18$. For realistic LH2 complexes, we need to consider different excitonic energies $E_{\alpha}$ from realizations of pigment energies $\varepsilon_{i}$ in order to describe the inhomogeneities (static disorder) arising from local protein configurations. Our choice of spectral density, nearestneighbor couplings, static disorder, geometry, and magnitude of the transition dipoles are justified by a previous independent analysis of experimental observations $[6,39,51,56-59]$, and when incorporated into our model, they reproduce observed absorption spectra and superradiance enhancement as shown in Figs. 2(b) and 2(c) (for details of calculations and parameters, see Appendixes D and E).

This parametrization of the $\mathrm{B} 850$ ring results in cooperative fluorescence from $\left\langle\sum_{\alpha} \chi_{\alpha} p_{\alpha}^{\text {th }}\right\rangle \approx 3$ pigments on average (where $\langle\cdot\rangle$ represents the average over static disorder), which is consistent with the experimental observations of superradiance in LH2 [39]. Moreover, fixing the decay rate for an isolated LH2 complex and the quantum yield of fluorescence to the experimentally observed values of $\Gamma_{\text {th }}=(1 \mathrm{~ns})^{-1}$ and $\phi=0.1$ allows us to obtain the distribution of decay rates $\Gamma_{\alpha}$ across the exciton manifold. A modest value of $r_{\mathrm{c}}=5 \AA$ for the correlation radius of the pigment's vibrational environments $[1,60]$ is sufficient to generate a significant redistribution of the dissipation rates to the higher-energy part of the excitonic manifold [Fig. 2(c)], as we would expect for an antiparallel arrangement of neighboring dipoles. The participation ratio shown in Fig. 2(c) exhibits a maximum $\left\langle\mathcal{P}_{\alpha}\right\rangle \approx 8$, also consistent with previous estimates [39], which underlie excitonic delocalization constrained to small portions of the B850 ring [Fig. 2(a)]. 
We should note that for realistic LH2 center-to-center distances $l$, the aggregation into arrays does not disrupt the excitonic manifold of single rings, as can be expected from the similarity of optical spectra of diluted and densely packed arrays [20]. Typical physiological conditions and lipid-reconstituted membranes exhibit a center-to-center distance $l \approx 8 \mathrm{~nm}$, with increasing intercomplex distances for larger lipid concentration [61-63]. On the other hand, the process of nanofabrication of LH2 arrays exploits hostguest interactions on a nano-imprinted substrate and does not involve lipids [20], which allows us to assume the 6.2-nm diameter of LH2 $\beta$ helices of Rb. sphaeroides [64] as the absolute minimum for $l$. Hence, it is reasonable to consider center-to-center separations of $l \gtrsim 6.5 \mathrm{~nm}$ in the nano-engineered arrays.

To assess the robustness of the single-ring excitonic manifold against the coherent interaction between neighboring LH2s, we proceed to diagonalize the full two-ring Hamiltonian $\left(\hat{H}^{(1)}+\hat{H}^{(2)}+\hat{V}^{(1,2)}\right)|\tilde{\mu}\rangle=E_{\tilde{\mu}}|\tilde{\mu}\rangle$ and present, in Fig. 2(c), the average over static disorder of the participation ratios, relative dipole strengths, and dissipation rates. Notice that even though the participation ratio in the LH2 pair $\mathcal{P}_{\tilde{\mu}}$ is slightly larger, very minor changes occur in the distributions of optical brightness $\chi_{\tilde{\mu}}$ and dissipation rates $\Gamma_{\tilde{\mu}}$ with respect to the single-ring eigenstates. As a consequence, optical absorption spectra are only slightly affected by the coherent interaction between LH2 rings [Fig. 2(b)]. This result confirms that the coherent electronic interaction for realistic values of $l$ does not significantly perturb the excitonic structure of isolated rings. The robustness of the single-ring excitonic manifold can be understood by noticing that the maximum coupling between any two LH2 excitons residing on different rings (averaged over static noise and relative rotations on coplanar rings) is below $40 \mathrm{~cm}^{-1}$ even for $l=6.5 \mathrm{~nm}$, which is much smaller than the nearestneighbor interactions within each ring, approximately $250-350 \mathrm{~cm}^{-1}$ [51,57]. One last argument in favor of the robustness of the single-ring excitonic manifold to the coherent coupling between rings is provided by looking at the residual inter-ring coherence after exciton thermalization. To do so, we set up a Lindblad equation describing the coherent interaction between two rings and local thermalization and dephasing,

$\partial_{t} \rho=-\frac{i}{\hbar}\left[\hat{H}^{(1)}+\hat{H}^{(2)}+\hat{V}^{(1,2)}, \rho\right]+\left(\mathcal{D}^{(1)}+\mathcal{D}^{(2)}\right) \rho$,

and solve for the steady state $\rho^{\text {ss }}$. Since both radiative and nonradiative decays take place on a much slower timescale, we neglect them here. The constant of proportionality between the thermalization rates $R_{\alpha \alpha^{\prime}}$ and the spectral density $\mathcal{J}\left(\left|E_{\alpha}-E_{\alpha^{\prime}}\right|\right)$ estimated via fluorescence linenarrowing experiments [60] is such that the approximately 200-fs timescale of equilibration in LH2 [56] is reproduced.
As we are interested in demonstrating that the stationary inter-ring coherence is typically negligible, we average the absolute value of the matrix elements $\left|\rho_{\alpha \beta}^{\text {ss }}\right|$ over static disorder to avoid ensemble dephasing. The results are shown in Fig. 2(d) for two inter-ring distances. Even for the shortest distance $(l=6.5 \mathrm{~nm})$, the average steady-state coherence $\left\langle\left|\rho_{\alpha \beta}^{\text {ss }}\right|\right\rangle$ between any two single-ring excitons $\left|\alpha_{1}\right\rangle$ and $\left|\beta_{2}\right\rangle$ is much smaller than $1 / 2$, which is the value it would take for a maximally coherent superposition of two states $\left(\left|\alpha_{1}\right\rangle+\left|\beta_{2}\right\rangle\right) / \sqrt{2}$.

At this point, we have established that for center-tocenter distances $l \geq 6.5 \mathrm{~nm}$, the incoherent energy transfer between neighboring B850 rings can be treated based upon the single-ring eigenstates. Now, we proceed to analyze the mechanisms that underlie this incoherent energy transfer between neighboring LH2s. In Fig. 2(e), we show how different single-ring excitons residing on two neighboring LH2 rings are connected via the average transfer rates $\left\langle W_{\alpha \beta}\right\rangle$. As we saw in the previous section, a shorter separation between unit cells leads to a substantial participation of optically dark states in the energy process, which can surpass the bright states in terms of transfer speed. In fact, also in this case, when reducing the distance $l$ from $8.5 \mathrm{~nm}$ to $6.5 \mathrm{~nm}$, the fastest transfer pathways (red dashed lines) shift from the low-energy part of the spectrum, where most of the dipole strength resides, to higher-energy excitons, which show larger delocalization. This finding underlines that the interaction in densely packed arrays does not depend on the exciton transition dipoles but rather on their delocalization, as quantified by the participation ratio.

\section{Nano-engineered LH2 arrays}

Armed with these facts, we now proceed to discuss the origin of the long-range diffusion observed in Ref. [20]. In this experiment, simultaneous excitation with a continuouswave diffraction-limited laser beam and imaging of the spatial profile of emission through confocal fluorescence detection enabled the readout of exciton propagation lengths of up to $2 \mu \mathrm{m}$ in quasi-1D assemblies of $\mathrm{LH} 2$ complexes. To the best of our knowledge, theoretical models could only explain such a diffusion by ignoring static disorder and underestimating dephasing $[6,65,66]$, resulting in long-range excitonic delocalization across approximately 40 pigments [42-44], a value that is in conflict with the experimental observations [39,45-48].

In order to examine this experiment, we determine the rates of the Pauli master equations, Eq. (3), for stochastic realizations of the pigment energies $\varepsilon_{i}$ and relative orientations of a 1D array of 1001 coplanar LH2 complexes, as shown schematically in Fig. 3(a), and study the stationary exciton distribution $\bar{p}_{\alpha}^{n}$. For moderate excitation power, which allows us to remain in the single excitation sector, driving can be modeled to take place on a single 
(a)

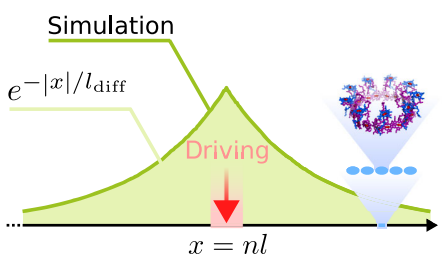

(b)

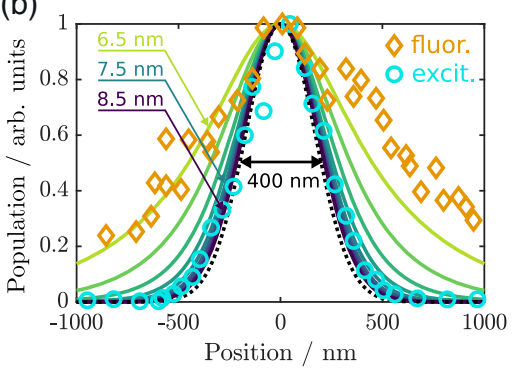

(c)

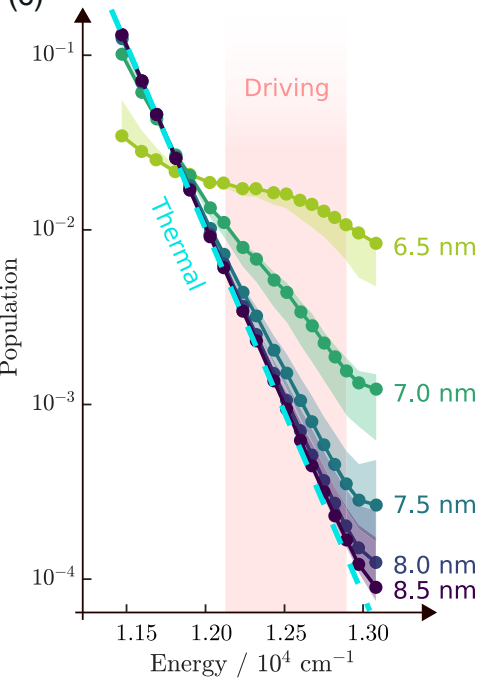

(d)

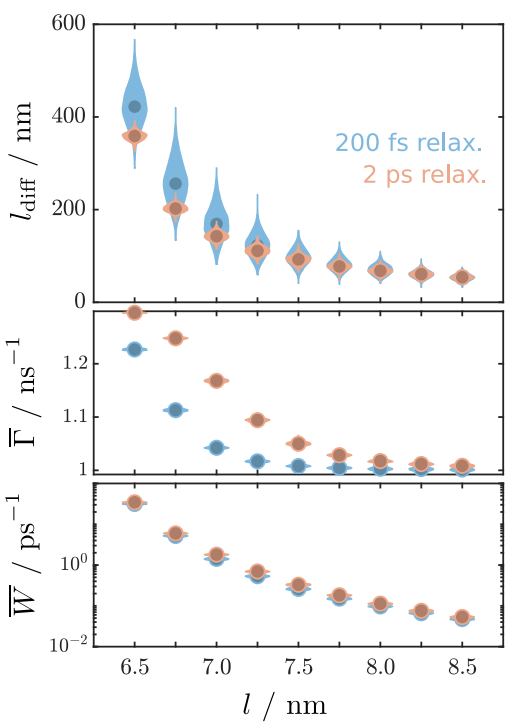

FIG. 3. Exciton propagation across a chain of LH2 units. (a) Energy transfer in a linear array of 1001 coplanar LH2 complexes subject to local driving results in an exponential population distribution. Intercomplex distances can vary between roughly $6.5 \mathrm{~nm}$ in nanoengineered arrays and $8.5 \mathrm{~nm}$ in biological membranes, resulting in inter-ring transfer rates $W_{\alpha \beta} \simeq(1-10 \mathrm{ps})^{-1}$ in typical physiological settings. Intracomplex dynamics is governed by interexciton dephasing and relaxation $R_{\alpha \alpha^{\prime}}$, with a typical timescale of $200 \mathrm{fs}$. (b) Spatial exciton distribution for distances $l$ ranging from $6.5 \mathrm{~nm}$ to $8.5 \mathrm{~nm}$ (green to violet lines), upon Gaussian driving with $400 \mathrm{~nm}$ full width at half maximum (black dotted line). The simulations for $l=6.5 \mathrm{~nm}$ agree with the experimental data taken from Ref. [20] (circles: excitation profile; diamonds: fluorescence profile). (c) Nonequilibrium energy distribution of exciton population averaged across the linear array for different distances $l$ (dots). The shaded areas represent the range in which the distribution varies when moving from the center to the ends of the array. The injection at the central ring takes place around $12500 \mathrm{~cm}^{-1}$ from the $\mathrm{B} 800$ ring (pink shaded area). At small $l$, the population deviates from the Boltzmann distribution (dashed line), and transfer effectively proceeds out of thermal equilibrium. (d) Distance dependence of effective diffusion length $l_{\text {diff }}$ (top), decay rate $\bar{\Gamma}$ (center), and transfer rate $\bar{W}$ (bottom). Their full distributions (patches) are shown together with their average (dots). LH2 arrays take advantage of fast local exciton thermalization (blue) to achieve longer-distance-albeit slower-energy transfer, compared to a hypothetical scenario with weaker coupling to local phonons (orange). All averages are performed over $10^{3}$ realizations of static disorder.

ring via incoherent B800-to-B850 energy transfer (Appendix F). Exciton distributions arising from a Gaussian laser profile can then be obtained by convolution of the driving profile with the solution for localized driving (Appendix A).

Despite the presence of static disorder and the multilevel structure of each unit cell, the stationary population distribution across the LH2 array is still characterized by an exponential distribution around the ring at which driving takes place [Fig. 3(a)]. Thus, we can characterize the population profile by a single parameter $l_{\text {diff }}$. When considering an initial Gaussian beam that is $400 \mathrm{~nm}$ wide (full width at half maximum), our model is able to reproduce the experimentally observed micron-range exciton propagation lengths if $l=6.5 \mathrm{~nm}$ [Fig. 3(b)], while natural distances of $8.0-8.5 \mathrm{~nm}$ yield a barely noticeable spread of the exciton density. This result suggests that the LH2 packing density is a key factor in determining the spatial extent of energy transfer. With a distance of $l=6.5 \mathrm{~nm}$, a competition between thermalization and transfer leads to the establishment of a nonequilibrium steady-state exciton population within antenna units $\bar{p}_{\alpha}$, which has a larger weight on highenergy dark states than the thermal distribution, as shown in
Fig. 3(c). This case is a clear signature that the nonequilibrium transfer across these arrays partially proceeds via high-energy dark states, which, as explained above, rely on excitonic delocalization within each ring unit cell.

Despite being optically dark, these high-energy excitons are more sensitive to nonradiative decay than their lowenergy bright counterpart, as shown in Fig. 2(c). Therefore, energy transfer and decay dynamics have competing effects on the diffusion length: Local exciton-phonon interactions leading to exciton thermalization slow down the transfer of excitons by moving population away from fast propagating high-energy states, while at the same time granting them more time to propagate, since low-energy excitons are less sensitive to nonradiative decay. Whether this leads to a larger diffusion length or not depends on the specifics of the system under consideration. In order to test this possibility, we artificially slow down the exciton thermalization timescale from $200 \mathrm{fs}$ to $2 \mathrm{ps}$, which corresponds, in practice, to forcing the steady-state exciton distribution in a given ring $\bar{p}_{\alpha}^{n}$ to be further away from thermal equilibrium $p_{\alpha}^{\text {th }}$. The shift of population towards the higher excitons results in a shorter-ranged transfer [Fig. 3(d), orange dots], revealing that these light-harvesting arrays operate in the generalized 
dark-state protection regime. The relevance of such a shelving mechanism is also confirmed by the behavior of the effective transfer and decay rates,

$$
\begin{gathered}
\bar{W}=\frac{1}{2}\left(\sum_{n, \alpha, \beta} W_{\alpha \beta}^{(n, n+1)} \bar{p}_{\beta}^{n+1}+\sum_{n, \alpha, \beta} W_{\beta \alpha}^{(n+1, n)} \bar{p}_{\alpha}^{n}\right), \\
\bar{\Gamma}=\sum_{n, \alpha} \Gamma_{\alpha}^{(n)} \bar{p}_{\alpha}^{n} .
\end{gathered}
$$

(Note that the presence of static disorder in the array forces us to keep track of the unit-cell index $n$ and to average over both forward and backward transfer rates.) For faster relaxation, i.e., larger coupling to local phonons, both effective transfer and decay rates are significantly decreased, as captured by Fig. 3(d).

As noticed recently [67], the transfer rate $\bar{W}$ benefits from the engagement of dark states at small inter-ring separations. Their participation allows for an increase in $\bar{W}$ that exceeds the one predicted by interactions only mediated by the collective dipoles $\mathbf{D}_{\alpha}^{(n)}$ and $\mathbf{D}_{\beta}^{(n+1)}$ of two neighboring $\mathrm{B} 850$ rings. The relevance of the finite size of unit cells naturally makes the transfer more dependent on their geometrical details and relative arrangement. Indeed, we notice, for example, that a systematic out-of-plane angle of just $5^{\circ}$ as observed in lipid-reconstituted membranes $[64,68]$, which slightly increases the distance between the closest pigments in neighboring rings, slows down the effective transfer rate and therefore decreases the diffusion length (Appendix F). However, the apparently beneficial involvement of fast-propagating dark states in the dynamics is countered by their sensitivity to nonradiative decay. Thus, in any situation in which we are interested in increasing the spatial extent of energy transfer rather than its speed, we need to consider the presence of realistic (radiative and nonradiative) decay channels and design the energy-transfer process accordingly. Closely packed LH2 arrays seem to naturally operate in a parameter regime that sustains a generalized dark-state protection mechanism, where fast exciton thermalization causes a shielding against nonradiative decay.

Finally, we notice from Fig. 3(d) that our model produces energy-transfer rates that are in excellent agreement with existing theoretical estimates obtained for larger interring separations $(l \gtrsim 7.5 \mathrm{~nm})$ [67,69], matching the conditions typically observed in reconstituted and biological light-harvesting membranes $[68,70]$. In this regime, intercomplex energy transfer proceeds from a completely equilibrated excitonic manifold [Fig. 3(c)], far from the nonequilibrium regime in which dark-state shelving becomes relevant. Thus, the design guidelines discussed in this work, while relevant for tightly packed nanoengineered systems, might be of secondary importance for more sparsely assembled biological LH2 membranes.

\section{CONCLUSIONS}

In conclusion, we have shown, with the help of an analytically solvable model, that room-temperature excitation energy transfer can benefit from quantum dynamics within modular unit cells, and we demonstrated that the resulting design principles apply in photosynthetic membranes with realistic physiological parameters, as well as in nanofabricated architectures. The resulting hybrid "quantum-classical" design can increase both the speed and propagation range thanks to the participation of the dark states due to excitonic delocalization within unit cells. On the one hand, improved speed can be achieved when the packing density of unit cells is made sufficiently large for the coupling of individual pigments on different unit cells to benefit from contributions of high-lying dark states. Crucially, close packing allows for exciton populations to depart from a thermal distribution, increasing the overall diffusion rate via nonequilibrium energy transfer. On the other hand, the exciton propagation length is extended by intraunit-cell relaxation, biasing electronic populations towards low-energy excitons, which are less sensitive to nonradiative decay and therefore increase the overall time window over which energy transfer can take place. This not only exemplifies the beneficial interplay of quantum coherent dynamics and environmental noise [71-73] but also provides basic mechanisms that underpin the micronrange propagation of excitations observed in artificial arrays of LH2 photosynthetic complexes. These results can be explained by the speedup of intercomplex transfer rates induced by dense packing of light-harvesting units and the protection from nonradiative decay provided by low-energy excitons. Although we do not expect this transfer mechanism to be at play in biological LH2 membranes due to their large intercomplex separations, it could be tested on nano-engineered platforms in experiments where the exciton diffusion length is measured for different light-harvesting arrays, prepared with different LH2 packing densities. Further corroborating evidence for the mechanism that we propose is derived from experimental observation of reduced exciton lifetimes in reconstituted LH2 membranes compared to isolated complexes [61]. Our model already captures this trend correctly in terms of an increased population of the high-energy excitons and thus could serve as a solid starting point for a more thorough quantitative analysis of this effect. While the multiscale model used in this work contains all the necessary elements to discuss general energy-transfer strategies in light-harvesting arrays, further improvements could be achieved by employing more refined theoretical descriptions of the light-harvesting units [74]. We plan to do so in the future by applying some recently developed numerical techniques, allowing for an exact treatment of the non-Markovian exciton dynamics [75].

The fact that the speed and spatial extent of energy transfer can be directly related to excitonic delocalization 
suggests the possibility of using partial delocalization restricted to single unit cells (due to the magnitude of noise in real room-temperature scenarios) as a resource to optimize the range of propagation of electronic excitations in technological applications with the goal of outperforming the already extremely high efficiency of natural photosynthesis. The general nature of these design principles hints that this energy-transfer scheme might find applications in a broad class of excitonic materials, not limited to the specific architecture explicitly discussed in this work, although it will be the task of future research to probe its technological feasibility.

\section{ACKNOWLEDGMENTS}

The authors are grateful to A. Block and N. van Hulst for useful discussions. This work was supported by the ERC Synergy grants BioQ and HyperQ, and an IQST PhD fellowship, and was made possible through the support of a grant from the John Templeton Foundation. The opinions expressed in this publication are those of the authors and do not necessarily reflect the views of the John Templeton Foundation. The authors acknowledge support by the state of Baden-Württemberg through bwHPC and the German Research Foundation (DFG) through Grant No. INST 40/ 575-1 FUGG (JUSTUS 2 cluster).

\section{APPENDIX A: EXACTLY SOLVABLE MODEL}

In this Appendix, we present the main steps leading to the analytical solution of the minimal model describing incoherent energy transfer across a linear array of dimerized unit cells, which locally support quantum delocalization. We start from Eq. (3) in the main text, i.e., the discrete diffusion equation of a linear array composed of dimerized unit cells, each hosting two levels, $\left|b_{n}\right\rangle$ and $\left|d_{n}\right\rangle$ (bright and dark), which can hop to the neighboring cells and are subject to intracell relaxation and fluorescence. Explicitly writing both the equations for the bright and dark components for local injection of excitations at site $n=0$, we obtain

$$
\begin{aligned}
\partial_{t} p_{b}^{n}= & W_{b b}\left(p_{b}^{n+1}+p_{b}^{n-1}-2 p_{b}^{n}\right)+W_{b d}\left(p_{d}^{n+1}+p_{d}^{n-1}\right) \\
& -2 W_{d b} p_{b}^{n}-R_{d b} p_{b}^{n}+R_{b d} p_{d}^{n}-\Gamma_{b} p_{b}^{n}+I_{b} \delta_{n, 0}, \\
\partial_{t} p_{d}^{n}= & W_{d d}\left(p_{d}^{n+1}+p_{d}^{n-1}-2 p_{d}^{n}\right)+W_{d b}\left(p_{b}^{n+1}+p_{b}^{n-1}\right) \\
& -2 W_{b d} p_{d}^{n}+R_{d b} p_{b}^{n}-R_{b d} p_{d}^{n}-\Gamma_{d} p_{d}^{n}+I_{d} \delta_{n, 0},
\end{aligned}
$$

where $p_{b}^{n}\left(p_{d}^{n}\right)$ denotes the population of the bright (dark) state of the $n$th dimer. The continuum limit is achieved by identifying $p_{\alpha}^{n}(t) / l=p_{\alpha}(x, t)(\alpha=b, d)$, with $x=n l$, and taking the interdimer separation $l$ to be vanishingly small so that $x$ becomes a continuous variable. To simplify the discussion, we assume that the cross-rates are equal, i.e., $W_{d b}=W_{b d}=w$, which is the case for the configuration assumed in the main text. If $W_{b d} \neq W_{d b}$, a drift term in the diffusion equation is introduced, and the final exciton distribution is not symmetric around the injection point $x=0$. Thus, we obtain two coupled diffusion equations:

$$
\begin{aligned}
\partial_{t} p_{b}(x, t)= & W_{b b} l^{2} \partial_{x}^{2} p_{b}(x, t)+w l^{2} \partial_{x}^{2} p_{d}(x, t)+I_{b} \delta(x) \\
& -\left(2 w+R_{d b}+\Gamma_{b}\right) p_{b}(x, t)+\left(2 w+R_{b d}\right) p_{d}(x, t),
\end{aligned}
$$

$$
\begin{aligned}
\partial_{t} p_{d}(x, t)= & w l^{2} \partial_{x}^{2} p_{b}(x, t)+W_{d d} l^{2} \partial_{x}^{2} p_{d}(x, t)+I_{d} \delta(x) \\
& +\left(2 w+R_{d b}\right) p_{b}(x, t) \\
& -\left(2 w+R_{b d}+\Gamma_{d}\right) p_{d}(x, t)
\end{aligned}
$$

Introducing the vectors $\mathrm{p}=\left(p_{b}, p_{d}\right)^{\top}, \mathbf{I}=\left(I_{b}, I_{d}\right)^{\top}$ and the matrices

$$
\begin{aligned}
& \mathrm{G}_{2}=\left(\begin{array}{cc}
W_{b b} & w \\
w & W_{d d}
\end{array}\right), \\
& \mathrm{G}_{0}=\left(\begin{array}{cc}
2 w+R_{d b}+\Gamma_{b} & -2 w-R_{b d} \\
-2 w-R_{d b} & 2 w+R_{b d}+\Gamma_{d}
\end{array}\right),
\end{aligned}
$$

we can rewrite Eqs. (A3) and (A4) more compactly as

$$
\partial_{t} \mathrm{p}(x, t)=\left(\mathrm{G}_{2} l^{2} \partial_{x}^{2}-\mathrm{G}_{0}\right) \mathrm{p}(x, t)+\mathrm{I} \delta(x) .
$$

Rewriting Eq. (A6) in terms of the Fourier transform $\hat{\mathrm{p}}(q, t)=\int \mathrm{d} x e^{-i q x} \mathrm{p}(x, t)$ and considering the stationary state at $t \rightarrow \infty$, we obtain the solution as

$\hat{\mathbf{p}}(q, \infty)=\frac{1}{\mathbf{G}_{0}+q^{2} l^{2} \mathbf{G}_{2}} \mathbf{I}=\sum_{k=0}^{\infty}\left(-q^{2} l^{2} \mathbf{G}_{0}^{-1} \mathbf{G}_{2}\right)^{k} \mathbf{G}_{0}^{-1} \mathbf{l}$.

The solution can be brought to a much more transparent form if we assume that the decay described by $\Gamma_{\alpha}$ takes place on a much slower timescale than interdimer and intradimer energy transfer. Within this approximation, which is typically satisfied in light-harvesting complexes, we have

$$
\mathrm{G}_{0}^{-1}=\frac{1}{\bar{\Gamma}}\left(\begin{array}{cc}
\bar{p}_{b} & \bar{p}_{b} \\
\bar{p}_{d} & \bar{p}_{d}
\end{array}\right)
$$

to leading order in $\Gamma_{\alpha}$, where $\bar{\Gamma}$ and $\bar{p}_{\alpha}$ are defined in Eqs. (9) and (10). This simple form allows us to sum the series in Eq. (A7) to

$$
\hat{\mathbf{p}}(q, \infty)=\frac{I_{b}+I_{d}}{\bar{\Gamma}} \frac{1}{1+q^{2} l^{2} \bar{W} / \bar{\Gamma}}\left(\begin{array}{c}
\bar{p}_{b} \\
\bar{p}_{d}
\end{array}\right),
$$

where $\bar{W}$ is defined in Eq. (8). Taking the inverse Fourier transform leads to the final form of the solution 


$$
\mathrm{p}(x, \infty)=\frac{I_{b}+I_{d}}{\bar{\Gamma}} \frac{1}{2 l_{\mathrm{diff}}} e^{-|x| / l_{\mathrm{diff}}}\left(\begin{array}{c}
\bar{p}_{b} \\
\bar{p}_{d}
\end{array}\right)
$$

which is the exciton distribution discussed in the main text. As one would expect, if interexciton conversion due to the cross-rates $R_{b d}, R_{d b}$, and $w$ is much faster than the decay timescale, both bright and dark manifolds have the same final distribution, differing only by the normalization constant $\bar{p}_{\alpha}$. It is easy to check that we would get the same exponential distribution if we considered a diffusion process involving unit cells containing a single level rather than two, with transfer and dissipation rates $\bar{W}$ and $\bar{\Gamma}$. Therefore, a dimeric unit cell results in the same type of diffusion as a monomeric one, where the large-scale diffusion properties can be tuned by changing the local dimer parameters.

A completely analogous procedure leads to the solution of the case in which $W_{b d} \neq W_{d b}$, which we do not present in detail here. The imbalance between these two rates does not change the definitions of $\bar{p}_{\alpha}$, $\bar{W}$, and $\bar{\Gamma}$ [which only depend on the average cross-rate $\left.w=\left(W_{b d}+W_{d b}\right) / 2\right]$ but leads to a drift coefficient proportional to $\Delta=\left(W_{b d}-W_{b d}\right)\left(\bar{p}_{b}-\bar{p}_{d}\right)$. As a final result, the exciton distribution is still exponentially localized around the injection point $x=0$, but the diffusion lengths for $x<0$ and $x>0$ are different. Thus, the diffusion is not symmetric. As a possible application, this property might be used to design excitonic wires that are able to switch the dominant direction of diffusion by small changes of their unit-cell properties, for example, implementing artificial photoprotection.

Although in the case of the exactly solvable model we consider a completely homogeneous system, it is possible to show that the presence of moderate static disorder does not modify the exponential shape of the stationary exciton distribution but only leads to a reduction of the diffusion length. This explains why, also in the case of the LH2 array presented in the main text, we still obtain an exponential distribution. To grasp the effects of static disorder, we consider a linear array of monomeric unit cells with some slight random inhomogeneity in the transfer rates. The transfer rate from site $n$ to site $n+1$ (and vice versa) is given by $W_{n}=\bar{W}+\delta W_{n}$, where we assume $\left\langle\delta W_{n}\right\rangle=0$ and $\left\langle\delta W_{n} \delta W_{m}\right\rangle=\sigma^{2} \delta_{n m}$. The average population distribution $\langle p(x)\rangle$ for small disorder can be obtained by expanding the solution in the form of Eq. (A7) to second order in $\delta W_{n}$ and taking the ensemble average. This process leads to an exponential average exciton distribution with diffusion length $l_{\text {diff }}^{\prime}=l_{\text {diff }}\left(1-\sigma^{2} / 2 \bar{W}^{2}\right)$, independent of the specific distribution of the rate fluctuations $\delta W_{n}$.

To conclude this section, we note that the stationary solution $\mathrm{p}(x, \infty)$ for local exciton injection $\delta(x)$ is sufficient to determine the exciton profile $\mathrm{p}^{\prime}(x, \infty)$ for any other (normalized) injection profile $g(x)$. In fact, defining $\hat{g}(q)$, the Fourier transform of $g(x)$, we immediately obtain that the new solution satisfies

$$
\hat{\mathbf{p}}^{\prime}(q, \infty)=\frac{\hat{g}(q)}{\mathbf{G}_{0}+q^{2} l^{2} \mathbf{G}_{2}} \mathbf{I}=\hat{\mathbf{p}}(q, \infty) \hat{g}(q) .
$$

Transforming back to real space, we obtain the new solution for generic driving as a convolution between the solution for local driving and the generic driving profile, namely,

$$
\mathbf{p}^{\prime}(x, \infty)=\int \mathrm{d} x^{\prime} \mathbf{p}\left(x^{\prime}, \infty\right) g\left(x-x^{\prime}\right) .
$$

The same principle allows us to draw conclusions for exciton propagation in a $\mathrm{LH} 2$ array upon driving with a Gaussian laser profile, using only the results of simulations for local driving.

\section{APPENDIX B: MICROSCOPIC ORIGIN OF NONRADIATIVE DECAY}

In this Appendix, we derive a microscopic Hamiltonian describing vibrationally induced nonradiative decay of a photoexcited molecular state. We start from the $a b$ initio Hamiltonian of a single chromophore. We determine the form of the nonadiabatic coupling at the basis of nonradiative decay in the usual harmonic approximation for intramolecular vibrations. We use this result to compute the internal conversion rate for delocalized excitonic states of a molecular aggregate.

\section{Molecular Hamiltonian}

The full Hamiltonian of a molecule is given by [50]

$$
\begin{aligned}
H(\mathbf{r}, \mathbf{p} ; \mathbf{R}, \mathbf{P})= & T_{\text {el }}(\mathbf{p})+V_{\text {el-el }}(\mathbf{r})+V_{\text {el-nuc }}(\mathbf{r} ; \mathbf{R}) \\
& +V_{\text {nuc-nuc }}(\mathbf{R})+T_{\text {nuc }}(\mathbf{P}),
\end{aligned}
$$

where $T$ denotes the kinetic energy and $V$ the Coulomb interactions. The sets of electronic coordinates and momenta are denoted by $\mathbf{r}$ and $\mathbf{p}$, whereas the nuclear degrees of freedom are described by $\mathbf{R}$ and $\mathbf{P}$. The typical challenge in condensed matter physics is to find approximate eigenstates and eigenvalues of this Hamiltonian. The huge difference between electronic and nuclear masses justifies the typical Born-Oppenheimer (BO) approach, where the electronic degrees of freedom are treated on a fully quantum level (i.e., $\mathbf{r} \rightarrow \hat{\mathbf{r}}$ and $\mathbf{p} \rightarrow \hat{\mathbf{p}}$ ), whereas the nuclear coordinates are kept fixed and their momenta are initially neglected. Thus, we can find the adiabatic electronic eigenstates by diagonalization of the electronic Hamiltonian at fixed $\mathbf{R}$ :

$$
\begin{aligned}
& {\left[T_{\text {el }}(\hat{\mathbf{p}})+V_{\text {el-el }}(\hat{\mathbf{r}})+V_{\text {el-nuc }}(\hat{\mathbf{r}} ; \mathbf{R})+V_{\text {nuc-nuc }}(\mathbf{R})\right]\left|\phi_{\mathbf{R}}^{i}\right\rangle} \\
& \quad=\varepsilon_{\mathbf{R}}^{i}\left|\phi_{\mathbf{R}}^{i}\right\rangle .
\end{aligned}
$$

The eigenstates $\left|\phi_{\mathbf{R}}^{i}\right\rangle$ and eigenenergies $\varepsilon_{\mathbf{R}}^{i}$-which are called potential energy surfaces (PES) - depend parametrically on the nuclear coordinates. Now, we express the 
Hamiltonian (B1) in terms of these adiabatic electronic states. Although their interpretation as physically relevant states depends on whether the nuclear kinetic energy is negligible or not, they always form a perfectly legitimate orthonormal basis of the electronic Hilbert space at fixed $\mathbf{R}$. Making use of Eq. (B2), we see that

$$
\left\langle\phi_{\mathbf{R}}^{i}|H(\hat{\mathbf{r}}, \hat{\mathbf{p}} ; \mathbf{R}, \mathbf{P})| \phi_{\mathbf{R}}^{j}\right\rangle=\delta^{i j} \varepsilon_{\mathbf{R}}^{i}+\left\langle\phi_{\mathbf{R}}^{i}\left|T_{\mathrm{nuc}}(\mathbf{P})\right| \phi_{\mathbf{R}}^{j}\right\rangle .
$$

Note that the second term on the right-hand side does not necessarily reduce to $\delta^{i j} T_{\text {nuc }}(\mathbf{P})$ because of the parametric dependence on $\mathbf{R}$ of the electronic states. However, if nuclei are considered as classical particles, the nuclear kinetic energy does not affect the electronic adiabatic wave function in any way, and the full molecular Hamiltonian (B1) takes the well-known BO form

$$
\hat{H}_{\mathrm{BO}}(\mathbf{R}, \mathbf{P})=\sum_{i}\left|\phi_{\mathbf{R}}^{i}\right\rangle\left\langle\phi_{\mathbf{R}}^{i}\right|\left(T_{\mathrm{nuc}}(\mathbf{P})+\varepsilon_{\mathbf{R}}^{i}\right) .
$$

The adiabaticity of this Hamiltonian is represented by the fact that the nuclear motion does not couple different electronic states: When the electrons are in the state $\left|\phi_{\mathbf{R}}^{i}\right\rangle$, the nuclei evolve according to the effective Hamiltonian $h_{i}(\mathbf{R}, \mathbf{P})=T_{\text {nuc }}(\mathbf{P})+\varepsilon_{\mathbf{R}}^{i}$.

Introducing a diagonal nuclear mass tensor $\mathbf{M}$ and expanding the $i$ th PES for small deviations from its stable equilibrium configuration $\mathbf{R}^{i}$ (i.e., close to its minimum $\left.\varepsilon_{\mathbf{R}^{i}}^{i}\right)$, the nuclear Hamiltonian $h_{i}$ becomes

$$
\begin{aligned}
h_{i}(\mathbf{R}, \mathbf{P}) & =\varepsilon_{\mathbf{R}^{i}}^{i}+\frac{1}{2}\left[\mathbf{P}^{\top} \mathbf{M}^{-1} \mathbf{P}+\left(\mathbf{R}-\mathbf{R}^{i}\right)^{\top} \mathrm{H}\left(\mathbf{R}-\mathbf{R}^{i}\right)\right] \\
& =\varepsilon_{\mathbf{R}^{i}}^{i}+\frac{1}{2}\left[\boldsymbol{\pi}^{\top} \boldsymbol{\pi}+\left(\boldsymbol{\xi}-\boldsymbol{\xi}^{i}\right)^{\top} \mathrm{D}\left(\boldsymbol{\xi}-\boldsymbol{\xi}^{i}\right)\right],
\end{aligned}
$$

where $\mathrm{H} \geq 0$ is the Hessian matrix of the $\operatorname{PES} \varepsilon_{\mathbf{R}}^{i}$ at $\mathbf{R}^{i}$. On the second line, we introduce the mass-rescaled normal coordinates and momenta $\boldsymbol{\xi}=\mathrm{UM}^{1 / 2} \mathbf{R}$ and $\boldsymbol{\pi}=\mathrm{UM}^{-1 / 2} \mathbf{P}$, where $U$ is the unitary transformation that diagonalizes the mass-rescaled Hessian, i.e., $\mathrm{U}^{\top} \mathrm{DU}=\mathrm{M}^{-1 / 2} \mathrm{HM}^{-1 / 2}$. Note that $\mathrm{D}$ has only diagonal entries, corresponding to the square of the normal frequencies $\omega_{k}^{2}$. As usual, we have neglected the Duschinsky mixing; that is, we have assumed that the Hessian $\mathrm{H}$ at the nuclear equilibrium configuration is independent of the specific PES, so it is diagonalized by the same transformation and yields the same eigenvalues (i.e., vibrational frequencies) for the PESs in which we are interested.

At this point, we can easily quantize the nuclear normal modes by redefining them in terms of a set of bosonic annihilation (creation) operators $\hat{b}_{k}\left(\hat{b}_{k}^{\dagger}\right)$. Assuming $\hbar=1$ from now on, we have

$$
\hat{\xi}_{k}=\frac{1}{\sqrt{2 \omega_{k}}}\left(\hat{b}_{k}+\hat{b}_{k}^{\dagger}\right),
$$

$$
\hat{\pi}_{k}=-i \sqrt{\frac{\omega_{k}}{2}}\left(\hat{b}_{k}-\hat{b}_{k}^{\dagger}\right)
$$

The quantized vibrational Hamiltonian (B5) becomes

$$
\hat{h}_{i}=\varepsilon_{\mathbf{R}^{i}}^{i}+\sum_{k} \omega_{k}\left[\left(\hat{b}_{k}-\sqrt{s_{k}^{i}}\right)^{\dagger}\left(\hat{b}_{k}-\sqrt{s_{k}^{i}}\right)+\frac{1}{2}\right] .
$$

Note that we have introduced the Huang-Rhys factors $s_{k}^{i}=\xi_{k}^{i 2} \omega_{k} / 2$, which, in general, depend on the PES under consideration.

Since our ultimate goal is to describe the dynamics of photoexcitations, we focus on two electronic states $\left|\phi_{\mathbf{R}}^{g}\right\rangle$ and $\left|\phi_{\mathbf{R}}^{e}\right\rangle$, describing the electronic ground state and the first optically excited state. We neglect any further dependence of these states on the nuclear coordinates and refer to them simply as $\left|\phi_{g}\right\rangle$ and $\left|\phi_{e}\right\rangle$. If we refer all energies and nuclear coordinates to the minimum of the PES of the electronic ground state, the BO Hamiltonian (B4) takes the usual spin-boson form with the pure dephasing interaction,

$\hat{H}_{\mathrm{BO}}=\varepsilon\left|\phi_{e}\right\rangle\left\langle\phi_{e}|+| \phi_{e}\right\rangle\left\langle\phi_{e}\right| \sum_{k} g_{k}\left(\hat{b}_{k}+\hat{b}_{k}^{\dagger}\right)+\sum_{k} \omega_{k} \hat{b}_{k}^{\dagger} \hat{b}_{k}$,

where $\varepsilon=\varepsilon_{\mathbf{R}^{e}}^{e}+\sum_{k} s_{k} \omega_{k}$ and $g_{k}=-\omega_{k} \sqrt{s_{k}^{e}}$. The adiabatic electron-phonon coupling can then be described by the spectral density

$$
\mathcal{J}(\omega)=\sum_{k} g_{k}^{2} \delta\left(\omega-\omega_{k}\right)
$$

\section{Nonadiabatic electron-phonon coupling}

Now, we return to Eq. (B3) and consider the effect that the nuclear kinetic energy has on the adiabatic electronic wave function. We take into account the quantum nature of nuclei as well, i.e., considering $\mathbf{R} \rightarrow \hat{\mathbf{R}}$ and $\mathbf{P} \rightarrow \hat{\mathbf{P}}$ as quantum mechanical operators. Note that the second term on the right-hand side of Eq. (B3) acts nontrivially on both electronic and nuclear degrees of freedom. To better understand this, we consider how the nuclear kinetic energy operator acts on a product state formed by $\left|\phi_{\mathbf{R}}^{j}\right\rangle$ and a nuclear state $\left|\chi_{\nu}\right\rangle$. In the coordinate representation, $\hat{\mathbf{P}}$ becomes the differential operator $-i \partial_{\mathbf{R}}$; therefore, we have

$$
\begin{aligned}
\left\langle\mathbf{r}, \mathbf{R}\left|T_{\mathrm{nuc}}(\hat{\mathbf{P}})\right| \phi_{\mathbf{R}}^{j}, \chi_{\nu}\right\rangle= & \sum_{k} \frac{\hat{P}_{k}^{2}}{2 M_{k}} \phi_{\mathbf{R}}^{j}(\mathbf{r}) \chi_{\nu}(\mathbf{R}) \\
= & \sum_{k} \frac{1}{2 M_{k}}\left[\phi_{\mathbf{R}}^{j}(\mathbf{r})\left(\hat{P}_{k}^{2} \chi_{\nu}(\mathbf{R})\right)\right. \\
& +2\left(\hat{P}_{k} \phi_{\mathbf{R}}^{j}(\mathbf{r})\right)\left(\hat{P}_{k} \chi_{\nu}(\mathbf{R})\right) \\
& \left.+\left(\hat{P}_{k}^{2} \phi_{\mathbf{R}}^{j}(\mathbf{r})\right) \chi_{\nu}(\mathbf{R})\right] .
\end{aligned}
$$


Using Eq. (B11), we can now compute the matrix element of the nuclear kinetic energy operator between two arbitrary electronic-vibrational states as

$$
\begin{aligned}
\left\langle\phi_{\mathbf{R}}^{i}, \chi_{\mu}\left|T_{\mathrm{nuc}}(\hat{\mathbf{P}})\right| \phi_{\mathbf{R}}^{j}, \chi_{\nu}\right\rangle= & \int \mathrm{d} \mathbf{r} \int \mathrm{d} \mathbf{R}\left\langle\phi_{\mathbf{R}}^{i}, \chi_{\mu} \mid \mathbf{r}, \mathbf{R}\right\rangle\left\langle\mathbf{r}, \mathbf{R}\left|T_{\mathrm{nuc}}(\hat{\mathbf{P}})\right| \phi_{\mathbf{R}}^{j}, \chi_{\nu}\right\rangle \\
= & \delta^{i j}\left\langle\chi_{\mu}\left|T_{\mathrm{nuc}}(\hat{\mathbf{P}})\right| \chi_{\nu}\right\rangle-i \sum_{k} \frac{1}{M_{k}} \int \mathrm{d} \mathbf{R}\left\langle\phi_{\mathbf{R}}^{i} \mid \partial_{R_{k}} \phi_{\mathbf{R}}^{j}\right\rangle \chi_{\mu}^{*}(\mathbf{R}) \hat{P}_{k} \chi_{\nu}(\mathbf{R}) \\
& -\sum_{k} \frac{1}{2 M_{k}} \int \mathrm{d} \mathbf{R}\left\langle\phi_{\mathbf{R}}^{i} \mid \partial_{R_{k}}^{2} \phi_{\mathbf{R}}^{j}\right\rangle \chi_{\mu}^{*}(\mathbf{R}) \chi_{\nu}(\mathbf{R})
\end{aligned}
$$

where the three terms appearing on the second line of Eq. (B12) directly originate from those appearing in Eq. (B11). It is easy to spot the first of them as the one giving rise to the $\mathrm{BO}$ Hamiltonian (B4). On the other hand, the terms involving derivatives of the adiabatic electronic wave function with respect to the nuclear coordinates are not necessarily proportional to $\delta^{i j}$, and therefore, they describe the nonadiabatic coupling between different PESs. Within the BO framework, they are neglected by the merits of the argument that the electronic wave functions depend very weakly on the nuclear coordinates; therefore, their derivatives are negligible. In fact, electronic wave functions usually vary significantly over distances of $1-10 \AA$, corresponding to typical internuclear separations. On the other hand, typical nuclei in organic molecules jiggle by about $0.01-0.1 \AA$ around their equilibrium position. On this length scale, therefore, electronic wave functions are expected to be fairly smooth and almost constant. Thus, the last two terms in Eq. (B12) can be regarded as higherorder contributions of a perturbative expansion whose zeroth-order term corresponds to the BO Hamiltonian.

For simplicity, let us focus only on the lowest nonzero order of this nonadiabatic perturbation, i.e., the one involving the first derivative of the adiabatic electronic wave function. Let us define the quantity $A_{k}^{i j}(\mathbf{R}):=$ $-i\left\langle\phi_{\mathbf{R}}^{i} \mid \partial_{R_{k}} \phi_{\mathbf{R}}^{j}\right\rangle$, which is closely related to the Berry connection. Note that we can always assume the phase of the adiabatic electronic wave functions to be independent of $\mathbf{R}$, corresponding to a specific gauge choice for the Berry connection. Together with the normalization condition $\left\langle\phi_{\mathbf{R}}^{i} \mid \phi_{\mathbf{R}}^{i}\right\rangle=1$, this immediately implies that $F_{k}^{i i}(\mathbf{R})=0$, meaning that this term only connects different electronic states and does not modify the vibrational Hamiltonian within a given PES. In addition, the orthogonality condition $\left\langle\phi_{\mathbf{R}}^{i} \mid \phi_{\mathbf{R}}^{j}\right\rangle=0$ further imposes a hermiticity constraint $A_{k}^{i j}(\mathbf{R})^{*}=A_{k}^{j i}(\mathbf{R})$. As usually assumed for optical transitions, we neglect the dependence of the coupling between different PESs on the nuclear configuration, which means that $A_{k}^{i j}(\mathbf{R}) \approx A_{k}^{i j}\left(\mathbf{R}^{i}\right)$ is practically constant in the integral over $\mathbf{R}$ in Eq. (B12). We also neglect any further dependence of the adiabatic states on the nuclear coordinates, as we did in the previous section in order to recast the $\mathrm{BO}$ Hamiltonian (B4) into spin-boson form (B9). Thus, we can finally write the initial molecular Hamiltonian (B1), including the lowestorder nonadiabatic correction as $\hat{H}_{\mathrm{BO}}+\hat{H}_{\text {nonad }}$, where

$$
\begin{aligned}
\left\langle\phi_{i}\left|\hat{H}_{\mathrm{nonad}}\right| \phi_{j}\right\rangle & =\sum_{k} A_{k}^{i j}\left(\mathbf{R}^{i}\right) \frac{\hat{P}_{k}}{M_{k}} \\
& =-i \sum_{k} \alpha_{k}^{i j} \sqrt{\frac{\omega_{k}}{2}}\left(\hat{b}_{k}-\hat{b}_{k}^{\dagger}\right),
\end{aligned}
$$

where, in the last step, we have rewritten the nuclear momenta in terms of the normal modes defined above and introduced the transformation $\boldsymbol{\alpha}^{i j}=\mathrm{UM}^{-1 / 2} \mathbf{A}^{i j}\left(\mathbf{R}^{i}\right)$ on the coefficients $A_{k}^{i j}\left(\mathbf{R}^{i}\right)$.

Thus, the nonadiabatic electron-phonon coupling can lead to transitions between different electronic states $\left|\phi_{i}\right\rangle$ and $\left|\phi_{j}\right\rangle$ initiated by the action of nuclear momenta. If we consider only two electronic states, namely, the ground state $g$ and one optically excited state $e$, we can see that it causes nonradiative transitions between $g$ and $e$ mediated by the coupling constant $f_{k}:=\alpha_{k}^{g e} \sqrt{\omega_{k} / 2}$. This process goes by the name of internal conversion (IC) and is therefore determined by the following spectral density:

$$
\mathcal{J}_{\text {nonad }}(\omega)=\sum_{k} f_{k}^{2} \delta\left(\omega-\omega_{k}\right),
$$

analogous to Eq. (B10).

\section{Internal conversion in molecular aggregates}

While it is generally true that the nonadiabatic couplings are much weaker than the pure-dephasing electron-phonon coupling, they still play a role on longer timescales, where other phenomena such as fluorescence come into play. Therefore, we should devise a way to treat IC on the same footing as fluorescence in molecular aggregates. We consider an aggregate of $N$ interacting two-level chromophores, each one described by the Hamiltonian (B13). We focus on the subspace spanned by the global ground state $|g\rangle=\left|\phi_{g}\right\rangle_{1} \ldots\left|\phi_{g}\right\rangle_{N}$ and the single excitations 
$|i\rangle=\left|\phi_{g}\right\rangle_{1} \ldots\left|\phi_{e}\right\rangle_{i} \ldots\left|\phi_{g}\right\rangle_{N}$. Assuming that the only sitedependent parameters of our model are the electronic excitation energies $\varepsilon_{i}$ and couplings $J_{i j}$, the Hamiltonian of the aggregate is

$$
\begin{aligned}
\hat{H}_{\mathrm{aggr}}= & \sum_{i} \varepsilon_{i}|i\rangle\left\langle i\left|+\sum_{i \neq j} J_{i j}\right| i\right\rangle\langle j|+\sum_{i} \sum_{k} \omega_{k} \hat{b}_{i k}^{\dagger} \hat{b}_{i k} \\
& +\sum_{i}|i\rangle\langle i| \sum_{k} g_{k}\left(\hat{b}_{i k}+\hat{b}_{i k}^{\dagger}\right) \\
& -i \sum_{i}(|g\rangle\langle i|+| i\rangle\langle g|) \sum_{k} f_{k}\left(\hat{b}_{i k}-\hat{b}_{i k}^{\dagger}\right) .
\end{aligned}
$$

On the first line, we recognize the free Hamiltonian of the excitonic system (diagonalized by excitons $|\alpha\rangle=$ $\sum_{i}|i\rangle\langle i \mid \alpha\rangle$ with energy $E_{\alpha}$ ) and the free Hamiltonian of environmental vibrations. On the second and third lines we find the system-bath interactions, mediated by the two bath operators

$$
\begin{gathered}
\hat{G}_{i}=\sum_{k} g_{k}\left(\hat{b}_{i k}+\hat{b}_{i k}^{\dagger}\right), \\
\hat{F}_{i}=-i \sum_{k} f_{k}\left(\hat{b}_{i k}-\hat{b}_{i k}^{\dagger}\right),
\end{gathered}
$$

which, respectively, cause pure dephasing in the site basis and nonradiative transitions between site excitations and the global ground state. Postponing a more detailed justification for later, we allow for correlations between different vibrational environments to be present when the bath is at equilibrium,

$$
\begin{aligned}
\left\langle\hat{G}_{i}(t) \hat{G}_{j}(0)\right\rangle_{\mathrm{eq}} & =\kappa_{i j} \mathcal{G}(t), \\
\left\langle\hat{F}_{i}(t) \hat{F}_{j}(0)\right\rangle_{\mathrm{eq}} & =\kappa_{i j} \mathcal{F}(t),
\end{aligned}
$$

where the time evolution is computed with respect to the free bath Hamiltonian and the expectation value $\langle\cdot\rangle_{\mathrm{eq}}$ is taken on the stationary state of the bath. The correlation functions $\mathcal{G}(t)$ and $\mathcal{F}(t)$ are assumed to be site independent and only depend on the temperature of the vibrational bath and on the spectral densities $\mathcal{J}(\omega)$ and $\mathcal{J}_{\text {nonad }}(\omega)$. We assume that the internal timescale of the bath is sufficiently fast and the system-bath coupling is sufficiently weak so that the dynamics of electronic excitations can be described by a Lindblad equation in the exciton basis.

We focus on internal conversion for now. Following the microscopic derivation outlined in Ref. [76], we obtain a Lindblad equation with jump operators $|g\rangle\langle\alpha|$ and rates

$$
\kappa_{\alpha} \Gamma_{\text {nonrad }}=\sum_{i, j}\langle\alpha \mid i\rangle \kappa_{i j}\langle j \mid \alpha\rangle \Gamma_{\text {nonrad }}
$$

appearing in Eq. (5). The rate $\Gamma_{\text {nonrad }}$ is the single-pigment nonradiative rate, determined by

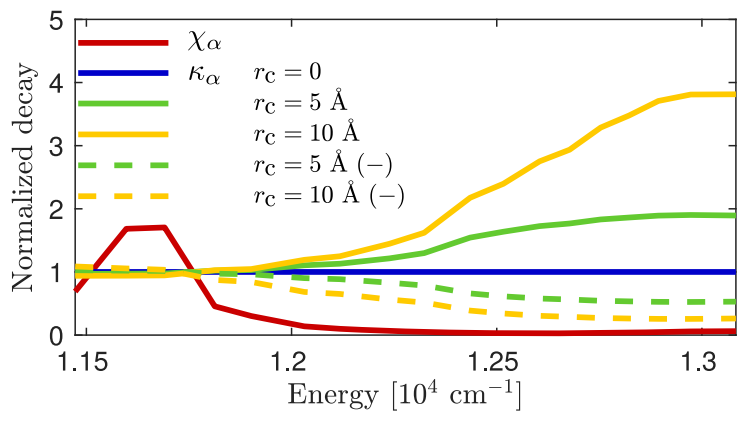

FIG. 4. Excitonic decay rates. Normalized decay rates from the excitonic manifold to the ground state for different processes in the $\mathrm{B} 850$ band of $\mathrm{LH} 2$ : radiative (red) and nonradiative with increasing bath correlation length (blue, green, and yellow solid lines). The dashed lines correspond to models with the same correlation length but assuming anticorrelated bath fluctuations for pigment couples that have opposite transition dipole moments. The decay rates are normalized such that, on average, they all give the same decay rate from a thermal exciton distribution. The results are obtained as ensemble averages over $10^{4}$ realizations of static disorder.

$\Gamma_{\text {nonrad }}=\int \mathrm{d} t e^{i \omega t} \mathcal{F}(t)=2 \pi \mathcal{J}_{\text {nonad }}(|\omega|)|n(\omega)+1|$.

In principle, it depends on the frequency at which the specific transition to the ground state takes place (i.e., $\omega=$ $E_{\alpha}$ for $\left.|g\rangle\langle\alpha|\right)$. However, since optical frequencies are much higher than vibrational and thermal energies, we can assume $\mathcal{J}_{\text {nonad }}(\omega)$ to be fairly small and constant across the excitonic energies $E_{\alpha}$.

The correlations introduced in Eqs. (B18) and (B19) are crucial for the determination of the distribution of the IC rate across the excitonic manifold, as shown in Fig. 4. For example, if the correlations are absent, i.e., $\kappa_{i j}=\delta_{i j}$, then Eq. (B21) predicts that the IC rate is essentially the same for all excitons (blue line). If, instead, we allow for some positive correlations decaying exponentially as a function of the distance between chromophores with some characteristic correlation length $r_{\mathrm{c}}$ (i.e., $\kappa_{i j}=e^{-r_{i j} / r_{\mathrm{c}}}$ ), excitons that delocalize over neighboring pigments show an increased decay rate (green and yellow solid lines). Correlations of such form are commonly used when modeling various optical spectra of pigment-protein complexes [60]. If the correlations are negative for neighboring pigments, the opposite behavior is observed (dashed lines). Optical correlations between transition dipole moments give the usual fluorescence profile (red line), with a few bright excitons at the bottom of the band. We will argue later in favor of the correlations between local vibrational environments that we have postulated by considering some experimental results.

\section{Other nonradiative decay pathways}

So far, we have seen how IC can be described microscopically and how it influences the nonradiative decay of 
different optical excitations in a molecular aggregate. However, there could be other processes that compete with fluorescence and IC on the same timescale, which could influence the effective decay rate from a given exciton. The other decay channel typically present in molecular systems is the intersystem crossing (ISC) [77]. During this process, a singlet excitation $S_{1}$ generated by optical absorption from the singlet ground state $S_{0}$ can be turned into an excited triplet $T_{1}$ by means of spin-orbit interactions. Since the radiative transition from $T_{1}$ to $S_{0}$ is strongly suppressed, the excitation is lost nonradiatively either by IC or by triplettriplet energy transfer to other pigments present in the molecular aggregates (i.e., carotenoids) before it can flip the spin of a neighboring oxygen molecule, thus forming singlet oxygen, which can cause oxidative damage to the photosynthetic apparatus.

If our focus is to follow the dynamics of excitons in a narrow band (i.e., the $\mathrm{B} 850$ of the $\mathrm{LH} 2$ complex), we do not want to take into account all of these processes: We are only interested in the rate at which spin-orbit interactions transfer excitations from one adiabatic PES to another. As we explicitly worked out for IC, it has been shown that the spin-orbit interaction also results in coupling between adiabatic PESs mediated by the nuclear momentum [78], which tells us that the effective Hamiltonian causing ISC will exhibit couplings of the same form as Eq. (B13), with properly redefined coupling constants. Therefore, the resulting ISC rate has the same form as Eq. (B21); i.e., it depends on the density of vibrational states at excitonic energies. Assuming that this density of states is sufficiently flat, as done before, in this case, we also see that the dependence of the rate on the excitonic state is dominated by the pattern of correlations between local baths. Therefore, ISC can be absorbed together with IC into a single rate $\kappa_{\alpha} \Gamma_{\text {nonrad }}$, describing the nonradiative decay of population from exciton $\alpha$.

\section{APPENDIX C: PHYSICAL ORIGIN OF CORRELATED ENVIRONMENTS}

In this Appendix, we argue in favor of the presence of correlations between the vibrational environments of single pigments belonging to the same pigment-protein complex, which take place on the timescale of nonradiative decay. First, we propose an estimate based on experimental results, and later, we move on to discuss a physical mechanism that can support intersite vibrational correlations, relying on intermolecular vibrational modes.

\section{Phenomenological view}

Let us step back for a moment from the microscopic derivation of internal conversion and follow another approach. We start from experimental evidence and use this knowledge to set up a phenomenological model of nonradiative decay of a molecular aggregate. The most
TABLE I. Single-pigment vs aggregate fluorescence. Quantum yield of fluorescence $(\phi)$ and decay lifetime $(\tau)$ of isolated Bchl- $a$ molecules and LH2 complexes, together with their radiative $\left(\Gamma_{\mathrm{rad}}\right)$ and nonradiative $\left(\Gamma_{\text {nonrad }}\right)$ decay rates (adapted from Ref. [39]).

\begin{tabular}{lcccc}
\hline \hline & $\phi(\%)$ & $\tau(\mathrm{ns})$ & $\Gamma_{\mathrm{rad}}\left(\mathrm{ns}^{-1}\right)$ & $\Gamma_{\text {nonrad }}\left(\mathrm{ns}^{-1}\right)$ \\
\hline Bchl- $a$ & 18 & 3.140 & 0.057 & 0.26 \\
LH2 & 9.86 & 0.986 & 0.10 & 0.91 \\
\hline \hline
\end{tabular}

valuable insight comes from comparing excited-state lifetimes $(\tau)$ and fluorescence quantum yields $(\phi)$ of isolated pigments with those of the aggregate. Table I shows theses quantities in the case of bacteriochlorophyll $a$ molecules (Bchl- $a$ ) and LH2 complexes [39]. Since the fluorescence quantum yield of LH2 is lower than the one of Bchl- $a$, there must be some additional decay channels in LH2 that are not present in the monomers and that can therefore be interpreted as an effect of aggregation.

To be more quantitative, let us define the quantum yield of fluorescence,

$$
\phi=\frac{\# \text { of photons emitted }}{\# \text { of photons absorbed }}=\frac{\Gamma_{\text {rad }}}{\Gamma_{\text {rad }}+\Gamma_{\text {nonrad }}}=\Gamma_{\text {rad }} \tau,
$$

and express the radiative and nonradiative decay rates as $\Gamma_{\text {rad }}=\phi / \tau$ and $\Gamma_{\text {nonrad }}=(1-\phi) / \tau$. The values reported in Ref. [39] allow us to determine radiative and nonradiative decay rates for Bchl- $a$ and LH2 (Table I). While the difference in radiative lifetimes between the monomer and the aggregate is easily explained by exciton delocalization and superradiance, a clear molecular mechanism underpinning the mismatch between nonradiative decay rates is not known with certainty. Nonetheless, we can put forward a simple argument. Since the nonradiative decay in LH2 is about 3.5 times faster than in Bchl, we can imagine the existence of additional dissipation channels. In fact, we expect the same intramolecular decay channels present in Bchl to be at play also in LH2. Thus, the additional dissipation present in LH2 must come from some other decay pathway that has no analogue in Bchl, which must account for $\left(\Gamma_{\text {nonrad }}^{\mathrm{LH} 2}-\Gamma_{\text {nonrad }}^{\mathrm{Bchl}}\right) / \Gamma_{\text {nonrad }}^{\mathrm{LH} 2}=71 \%$ of the nonradiative dissipation. The first reasonable candidate is the protein environment, which can offer other IC pathways through vibrations and conformational changes. Since the B850 ring of LH2 is composed of dimeric subunits bound to the same protein, it makes sense to assume that the IC channels offered by the protein can, in principle, be correlated. Following this argument, we allow for correlations between the vibrational environments that couple to IC and ISC transitions. As a consequence, excitons that delocalize on neighboring pigments can experience a modified decay rate, as discussed above and shown in Fig. 4. 


\section{Microscopic mechanism}

We have seen how, within a BO framework, a coupling arises between (optical) electronic transitions and vibrations of a molecule. A sudden change in the electronic state leads to a different electrostatic potential experienced by the nuclei, which therefore initiate their dynamics. For this reason, this coupling involves only vibrations of the nuclei over which the electronic states are delocalized. It is therefore clear that intramolecular modes will experience this type of direct coupling to the electronic dynamics. However, if the chromophore is embedded in a protein environment, the vibrational modes of the protein will also influence electronic energies, leading to a direct coupling between electronic excitations of the chromophore and longer wavelength vibrational modes. This observation lies at the heart of theoretical descriptions of electronic resonances coupled to a shared phonon environment [79]. Thus, the pure dephasing coupling in Eq. (B15) can be written explicitly in terms of these protein vibrational modes $\hat{c}_{q}$ with frequency $\nu_{q}$ as

$\hat{H}_{\mathrm{SB}}=\sum_{i}|i\rangle\langle i|\left[\sum_{k} g_{k}\left(\hat{b}_{i k}+\hat{b}_{i k}^{\dagger}\right)+\sum_{q} \xi_{i q}\left(\hat{c}_{q}+\hat{c}_{q}^{\dagger}\right)\right]$.

By redefining the operators $\hat{G}_{i}$ in Eq. (B16) to also include the protein modes $\hat{c}_{q}$, we obtain the following bath correlation functions,

$$
\begin{aligned}
\left\langle\hat{G}_{i}(t) \hat{G}_{j}(0)\right\rangle_{\mathrm{eq}}= & \sum_{k} g_{k}^{2}\left[e^{-i \omega_{k} t}\left(n\left(\omega_{k}\right)+1\right)+e^{i \omega_{k} t} n\left(\omega_{k}\right)\right] \delta_{i j} \\
& +\sum_{q} \xi_{i q} \xi_{j q}\left[e^{-i \nu_{q} t}\left(n\left(\nu_{q}\right)+1\right)+e^{i \nu_{q} t} n\left(\nu_{q}\right)\right] .
\end{aligned}
$$

While the term on the first line (corresponding to intramolecular modes) vanishes for $i \neq j$, the second term (arising from protein modes) is able to generate correlations between sites. Moreover, since protein motion takes place on a larger and slower scale than intramolecular vibrations, it is reasonable to assume that two neighboring pigments $i$ and $j$ couple to the protein motion with the same phase $\left(\xi_{i q} \xi_{j q}>0\right)$. This process results in positive intersite correlations and thus can lead to the redistribution of the nonradiative decay rates discussed in Appendix B.

Another possible mechanism able to generate positive intersite vibrational correlations is based on the idea that the vibrational motion of a chromophore can mechanically couple to the slow vibrations of the protein environment. To better formalize this idea, let us consider a slightly modified version of the model described by the Hamiltonian (B15). To simplify the discussion, we consider a molecular aggregate composed only of two pigments, i.e., $i=1,2$, (a)

Same orientation
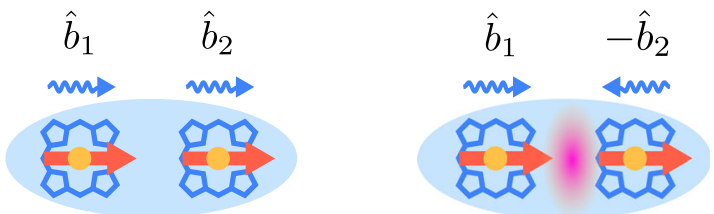

(b)
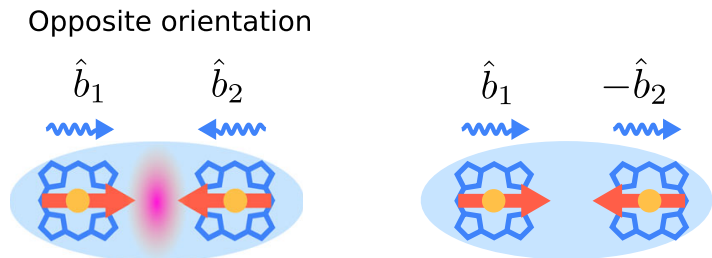

FIG. 5. Collective modes in a molecular dimer. (a) If the two molecules immersed in an elastic medium (roughly modeling the protein environment) have the same orientation, only the antisymmetric superposition of local vibrational modes causes stretching or compression of the medium. (b) If the molecules have opposite orientation (like two neighboring B850 pigments in LH2), the symmetric superposition of local modes causes stretching or compression of the medium.

and we neglect the direct coupling between electronic excitations and protein modes [Eq. (C1)]. If the two pigments are bound to the same protein, it makes sense to assume that their intramolecular modes $\hat{b}_{1, k}$ and $\hat{b}_{2, k}$ couple to a set of common modes $\hat{c}_{q}$ with frequency $\nu_{q}$. Since these shared modes are associated with the protein structure, it is reasonable to assume that they are much slower and effectively classical, meaning that their energy $\nu_{q}$ is much smaller than thermal energy $k_{\mathrm{B}} T$. We assume that the intramolecular modes of the two pigments couple the protein vibrations with the same strength and phase. This choice makes sense if we think that the two pigments have opposite orientation and are embedded in an elastic medium (see Fig. 5). The Hamiltonian of the vibrational bath then reads

$$
\begin{aligned}
\hat{H}_{\mathrm{B}}= & \sum_{k} \omega_{k}\left(\hat{b}_{1, k}^{\dagger} \hat{b}_{1, k}+\hat{b}_{2, k}^{\dagger} \hat{b}_{2, k}\right)+\sum_{q} \nu_{q} \hat{c}_{q}^{\dagger} \hat{c}_{q} \\
& +\sum_{k, q} \eta_{k q}\left(\hat{b}_{1, k} \hat{c}_{q}^{\dagger}+\hat{b}_{2, k} \hat{c}_{q}^{\dagger}+\text { H.c. }\right),
\end{aligned}
$$

where we have neglected the counterrotating coupling terms to simplify the following treatment, although they could be included. Defining the symmetric and antisymmetric combinations of intramolecular modes $\hat{b}_{k}=\left(\hat{b}_{1, k}+\hat{b}_{2, k}\right) / \sqrt{2}$ and $\hat{b}_{k}^{-}=\left(\hat{b}_{1, k}-\hat{b}_{2, k}\right) / \sqrt{2}$, we can rewrite the coupling as $\sum_{k, q} \sqrt{2} \eta_{k q} \hat{b}_{k} \hat{c}_{q}^{\dagger}+$ H.c.; i.e., the antisymmetric modes $\hat{b}_{k}^{-}$are insensitive to the coupling to the protein. The symmetric modes, on the other hand, mix with the protein vibrations, yielding the new set of normal modes 


$$
\begin{aligned}
& \hat{\tilde{b}}_{k}=\sum_{k^{\prime}} \mathrm{U}_{k k^{\prime}} \hat{b}_{k^{\prime}}+\sum_{q} \mathrm{v}_{k q} \hat{c}_{q}, \\
& \hat{\tilde{c}}_{q}=\sum_{k} \mathrm{u}_{q k} \hat{b}_{k}+\sum_{q^{\prime}} \mathrm{V}_{q q^{\prime}} \hat{c}_{q^{\prime}},
\end{aligned}
$$

with frequencies $\tilde{\omega}_{k}$ and $\tilde{\nu}_{q}$. Thanks to its unitarity, the transformation can be easily inverted, and the bath operators $\hat{G}_{i}$ defined in Eq. (B16) can be reexpressed as

$$
\begin{aligned}
\hat{G}_{1(2)} & =\frac{1}{\sqrt{2}}\left(\sum_{k} g_{k} \hat{b}_{k} \pm \sum_{k} g_{k} \hat{b}_{k}^{-}\right)+\text {H.c. } \\
& =\frac{1}{\sqrt{2}}\left(\sum_{k} \mathrm{G}_{k} \hat{\tilde{b}}_{k}+\sum_{q} \mathrm{~g}_{q} \hat{\tilde{c}}_{q} \pm \sum_{k} g_{k} \hat{b}_{k}^{-}\right)+\text {H.c. }
\end{aligned}
$$

where we have defined the transformed system-bath couplings

$$
\mathbf{G}_{k}=\sum_{k^{\prime}} g_{k^{\prime}} \mathbf{U}_{k^{\prime} k}^{*} \quad \text { and } \quad g_{q}=\sum_{k^{\prime}} g_{k^{\prime}} \mathbf{u}_{k^{\prime} q}^{*}
$$

Analogous expressions can be found for the operators $\hat{F}_{i}$ defined in Eq. (B17).

Let us now focus on the bath correlation functions for the pure dephasing environment introduced in Eq. (B18), evaluated on the thermal state $\rho_{\text {eq }} \propto e^{-\hat{H}_{\mathrm{B}} / k_{\mathrm{B}} T}$. (The system-bath coupling leading to internal conversion can be treated in a completely analogous way.) Using the new normal-mode decomposition of $\hat{G}_{i}$ [Eq. (C6)], we get

$$
\begin{aligned}
\left\langle\hat{G}_{1}(t) \hat{G}_{1(2)}(0)\right\rangle_{\mathrm{eq}} & \\
= & \frac{1}{2} \sum_{k} \mathrm{G}_{k}^{2}\left[e^{-i \tilde{\omega}_{k} t}\left(n\left(\tilde{\omega}_{k}\right)+1\right)+e^{i \tilde{\omega}_{k} t} n\left(\tilde{\omega}_{k}\right)\right] \\
& +\frac{1}{2} \sum_{q} g_{q}^{2}\left[e^{-i \tilde{\nu}_{k} t}\left(n\left(\tilde{\nu}_{q}\right)+1\right)+e^{i \tilde{\nu}_{q} t} n\left(\tilde{\nu}_{q}\right)\right] \\
& \pm \frac{1}{2} \sum_{k} g_{k}^{2}\left[e^{-i \omega_{k} t}\left(n\left(\omega_{k}\right)+1\right)+e^{i \omega_{k} t} n\left(\omega_{k}\right)\right]
\end{aligned}
$$

To lowest order in the intersite vibrational coupling $\eta_{k q}$, we find the following perturbative expressions

$$
\begin{gathered}
\mathrm{G}_{k} \approx g_{k}, \quad \mathrm{~g}_{q} \approx-\sum_{k} \frac{\sqrt{2} g_{k} \eta_{k q}}{\omega_{k}-\nu_{q}}, \\
\tilde{\omega}_{k} \approx \omega_{k}+\sum_{q} \frac{2 \eta_{k q}^{2}}{\omega_{k}-\nu_{q}}, \quad \tilde{\nu}_{q} \approx \nu_{q}-\sum_{k} \frac{2 \eta_{k q}^{2}}{\omega_{k}-\nu_{q}} .
\end{gathered}
$$

Note that, in order to be perfectly consistent, we should also keep the second-order correction to $\mathrm{G}_{k}$. However, since the protein modes generally have much smaller energies $\nu_{q}$ than both $k_{\mathrm{B}} T$ and intramolecular modes $\omega_{k}$, their thermal occupation number is much higher, i.e., $n\left(\tilde{\nu}_{q}\right) \gg n\left(\tilde{\omega}_{k}\right)$. Therefore, the correction arising from the first line in Eq. (C8) is negligible with respect to the one originating from the second line. Taking into account all of these assumptions, we obtain

$$
\begin{aligned}
\left\langle\hat{G}_{1}(t) \hat{G}_{1}(0)\right\rangle_{\mathrm{eq}} & \approx \sum_{k} g_{k}^{2}\left[e^{-i \omega_{k} t}\left(n\left(\omega_{k}\right)+1\right)+e^{i \omega_{k} t} n\left(\omega_{k}\right)\right], \\
\left\langle\hat{G}_{1}(t) \hat{G}_{2}(0)\right\rangle_{\mathrm{eq}} \approx & \sum_{q}\left(\sum_{k} \frac{g_{k} \eta_{k q}}{\omega_{k}-\nu_{q}}\right)^{2} \\
& \times\left[e^{-i \nu_{q} t}\left(n\left(\nu_{q}\right)+1\right)+e^{i \nu_{q} t} n\left(\nu_{q}\right)\right] . \quad(\mathrm{C} 12
\end{aligned}
$$

Note that both of these equations can be rewritten in terms of positive spectral densities: In the case of Eq. (C11), we use the one defined in Eq. (B10), whereas for Eq. (C12), we can define

$$
\Delta \mathcal{J}(\omega)=\sum_{q}\left(\sum_{k} \frac{g_{k} \eta_{k q}}{\omega_{k}-\nu_{q}}\right)^{2} \delta\left(\omega-\nu_{q}\right) .
$$

Taking the Fourier transform of these correlation functions, we obtain the rates that allow us to write down a Lindblad equation for the reduced dynamics of the excitons, which are proportional to the spectral densities $\mathcal{J}(\omega)$ and $\Delta \mathcal{J}(\omega)$.

At this point, we can see that our particular choice of coupling the local intramolecular modes to the common protein modes with the same phase leads to the establishment of positive intersite correlations $[\Delta \mathcal{J}(\omega)>0]$. If we choose to couple local modes with the opposite phase instead, we would end up with negative correlations between the vibrational environments.

\section{APPENDIX D: LINE SHAPES, LINEAR SPECTRA, AND TRANSFER RATES}

In this section, we determine the line shapes of an excitonic system (i.e., our unit cells), which allow for the calculation of both linear optical spectra and excitonic transfer rates. Consider an excitonic system with Hamiltonian $\hat{H}=\sum_{\alpha} E_{\alpha}|\alpha\rangle\langle\alpha|$, which we identify as our unit cell, where Greek letters denote exciton states of the unit cell $|\alpha\rangle=\sum_{i}|i\rangle\langle i \mid \alpha\rangle$. (Here, we relax the convention used in the main text, according to which primed indices refer to the same subunit.) Within a single unit cell, excitedstate population thermalizes because of the interaction between electronic and vibrational degrees of freedom of both intramolecular and intermolecular origin. The main electron-phonon coupling mechanism is described by the pure-dephasing interaction presented in the aggregate Hamiltonian (B15). Under the conditions of weak 
electron-phonon coupling and fast vibrational relaxation, this process can be described by a Lindblad equation. In the presence of correlations $\kappa_{i j}$ between different local environments, we can write down the resulting Lindblad dissipator as

$$
\begin{aligned}
\mathcal{D} \rho= & \sum_{\alpha \neq \beta} R_{\alpha \beta}\left(|\alpha\rangle\langle\beta|\rho| \beta\rangle\langle\alpha|-\frac{1}{2}\{|\beta\rangle\langle\beta|, \rho\}\right) \\
& +2 \gamma \sum_{\alpha, \beta} \zeta_{\alpha \beta}\left(|\alpha\rangle\langle\alpha|\rho| \beta\rangle\langle\beta|-\frac{1}{2} \delta_{\alpha \beta}\{|\alpha\rangle\langle\alpha|, \rho\}\right),
\end{aligned}
$$

where the first line describes population transfer across different excitons and the second describes pure dephasing processes. The rates can be obtained, respectively, as

$$
\begin{gathered}
R_{\alpha \beta}=2 \pi \mathcal{J}\left(\left|\omega_{\alpha \beta}\right|\right)\left|n\left(\omega_{\alpha \beta}\right)\right| \sum_{i, j}\langle\alpha \mid i\rangle\langle i \mid \beta\rangle \kappa_{i j}\langle\beta \mid j\rangle\langle j \mid \alpha\rangle, \\
2 \gamma \zeta_{\alpha \beta}=2 \gamma \sum_{i, j}\langle\alpha \mid i\rangle\langle i \mid \alpha\rangle \kappa_{i j}\langle\beta \mid j\rangle\langle j \mid \beta\rangle,
\end{gathered}
$$

where $\omega_{\alpha \beta}=E_{\alpha}-E_{\beta}$, and $\gamma$ is the single-pigment optical dephasing rate. Note that, when the local vibrational environments are not correlated, i.e., $\kappa_{i j}=\delta_{i j}$, the factors involving excitonic amplitudes in Eqs. (D2) and (D3) reduce to the spatial overlap between excitons and their participation ratio. Lindblad dynamics in the exciton basis results in an exponential decay of the optical coherences between exciton $\alpha$ and the ground state $g$ with a rate $\gamma_{\alpha}=\gamma \zeta_{\alpha \alpha}+\sum_{\beta(\neq \alpha)} R_{\beta \alpha} / 2$, whereas the interexciton coherence decays with a rate $\gamma_{\alpha \beta}=\gamma_{\alpha}+\gamma_{\beta}-2 \gamma \zeta_{\alpha \beta}$. This result leads to simple expressions when computing optical absorption and emission spectra. These expressions are related to the absorption and emission tensors, $A(\omega)$ and $E(\omega)$, respectively, defined by their matrix elements

$$
\begin{aligned}
& \mathrm{A}_{\alpha \beta}(\omega)=\int \mathrm{d} t e^{i \omega t}\left\langle\hat{\sigma}_{\alpha}(t) \hat{\sigma}_{\beta}^{\dagger}(0)\right\rangle_{g}, \\
& \mathrm{E}_{\alpha \beta}(\omega)=\int \mathrm{d} t e^{-i \omega t}\left\langle\hat{\sigma}_{\beta}^{\dagger}(t) \hat{\sigma}_{\alpha}(0)\right\rangle_{e} .
\end{aligned}
$$

Here, $\hat{\sigma}_{\mu}(t)$ denotes the Heisenberg time evolution of the annihilation operator of exciton $\mu, \hat{\sigma}_{\mu}=|g\rangle\langle\mu|$, and $\langle\cdot\rangle_{g(e)}$ denotes the average over the equilibrium ground (excited) state. Evolving the transition dipole operator through the dual of $\mathcal{D}$ results in the simple expression $\hat{\sigma}_{\mu}(t)=|g\rangle\langle\mu| e^{-\left(\gamma_{\mu}+i E_{\mu}\right) t}$, which leads to diagonal absorption and emission tensors, with each exciton having a Lorentzian line shape, i.e., $\mathrm{A}_{\alpha \beta}(\omega)=\delta_{\alpha \beta} f_{\alpha}(\omega)$ and $\mathrm{E}_{\alpha \beta}(\omega)=\delta_{\alpha \beta} f_{\alpha}(\omega) p_{\alpha}^{\text {th }}$, where $p_{\alpha}^{\text {th }}$ is the thermal population of exciton $\alpha$, and

$$
f_{\alpha}(\omega)=\frac{2 \gamma_{\alpha}}{\gamma_{\alpha}^{2}+\left(\omega-E_{\alpha}\right)^{2}} .
$$

Once we have the line shape for each exciton, we weight each individual line shape by its associated brightness $\chi_{\alpha} \approx\left|\mathbf{D}_{\alpha}\right|^{2} / d^{2}$ and straightforwardly calculate the absorption spectrum of the unit cell as $\omega \sum_{\alpha} \chi_{\alpha} f_{\alpha}(\omega)$. In the case of the B850 subunit, once averaged over static disorder, this expression gives excellent agreement with experimental results, as shown in the main text [Fig. 2(b)].

If we introduce a second excitonic system, weakly interacting (with respect to the timescales associated with dephasing) with the first one via dipole-dipole couplings

$V_{i i^{\prime}}=\left\langle i|\hat{V}| i^{\prime}\right\rangle=\frac{1}{4 \pi \varepsilon_{0}} \frac{d^{2}}{r_{i i^{\prime}}^{3}}\left[\mathbf{e}_{i} \cdot \mathbf{e}_{i^{\prime}}-3\left(\mathbf{e}_{i} \cdot \mathbf{n}_{i i^{\prime}}\right)\left(\mathbf{e}_{i^{\prime}} \cdot \mathbf{n}_{i i^{\prime}}\right)\right]$,

we can calculate the incoherent energy-transfer rate between excitons $\alpha$ and $\alpha^{\prime}$ on the two subunits via generalized Förster theory, according to which, we have

$$
\begin{aligned}
W_{\alpha \alpha^{\prime}} & =\frac{\left|V_{\alpha \alpha^{\prime}}\right|^{2}}{2 \pi} \int \mathrm{d} \omega f_{\alpha}(\omega) f_{\alpha^{\prime}}(\omega) \\
& =2\left|V_{\alpha \alpha^{\prime}}\right|^{2} \frac{\gamma_{\alpha \alpha^{\prime}}}{\omega_{\alpha \alpha^{\prime}}^{2}+\gamma_{\alpha \alpha^{\prime}}^{2}}
\end{aligned}
$$

where $V_{\alpha \alpha^{\prime}}=\sum_{i, i^{\prime}}\langle\alpha \mid i\rangle V_{i i^{\prime}}\left\langle i^{\prime} \mid \alpha^{\prime}\right\rangle$, which reduces to Eq. (4) from the main text in the case of dimeric unit cells, once $\hbar$ is reintroduced.

\section{APPENDIX E: PARAMETRIZATION OF THE B850 RING}

Figure 6 and Table II summarize the geometry and the parameters that have been used in the simulations of the B850 rings. The ring structure is dimerized, meaning that each pigment $i$ is identified by two indices, one specifying the dimer $(n=1, \ldots, 9)$ and the other the position within the dimer $(\nu=1,2)$. The two bacteriochlorophyll (Bchl) molecules belonging to the same dimer are usually labeled $\alpha$ and $\beta$. A single ring is described by the Hamiltonian

$$
\begin{aligned}
\hat{H}= & \sum_{i}\left(\varepsilon+\delta \varepsilon_{i}\right)|i\rangle\left\langle i\left|+\sum_{i \neq j} J_{i j}\right| i\right\rangle\langle j| \\
= & \sum_{n=1}^{9}\left[\left(\varepsilon+\delta \varepsilon_{n, 1}\right)|n, 1\rangle\left\langle n, 1\left|+\left(\varepsilon+\delta \varepsilon_{n, 2}\right)\right| n, 2\right\rangle\langle n, 2|\right. \\
& \left.+J_{1}(|n, 1\rangle\langle n, 2|+\text { H.c. })+J_{2}(|n, 2\rangle\langle n+1,1|+\text { H.c. })\right] \\
& +\sum_{i, j}{ }^{\prime} J_{i j}|i\rangle\langle j|,
\end{aligned}
$$

where the primed sum indicates summation over all couples of nonadjacent pigments and $J_{i j}$ stands, in this case, for the dipole-dipole interaction between pigments. 


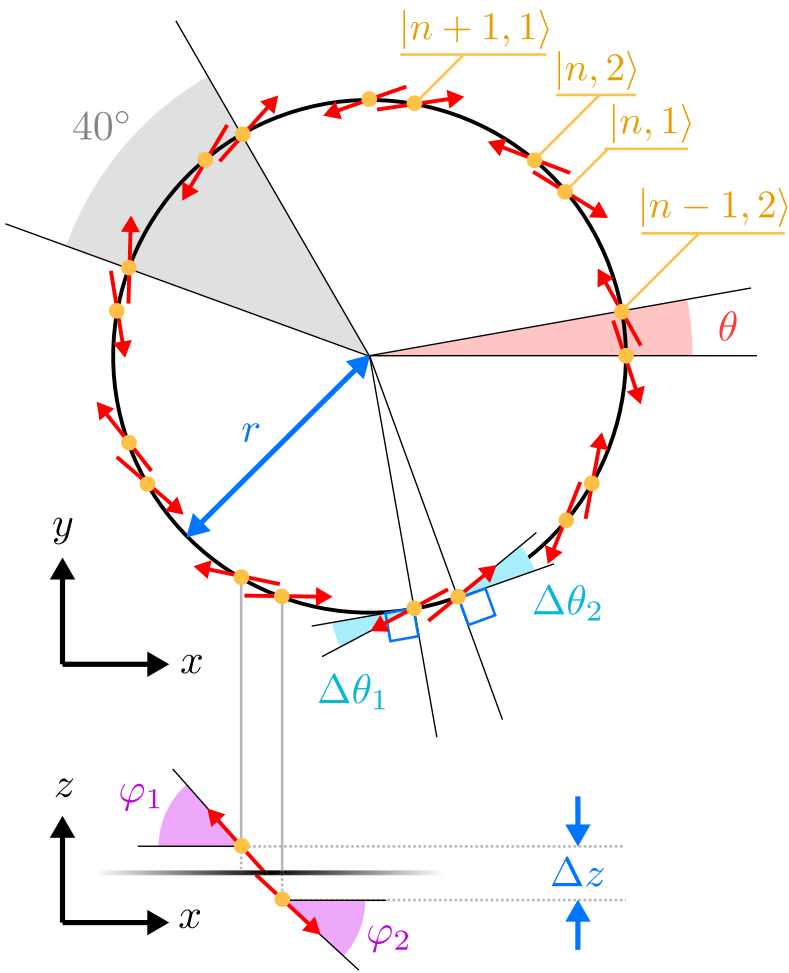

FIG. 6. Geometry of a B850 ring. We show projections on the $x y$ plane (top) and on the $x z$ plane (bottom) of a B850 ring lying on the $x y$ plane. The $z$ direction is magnified by a factor 10 , and the angles $\varphi_{1}$ and $\varphi_{2}$ are rescaled accordingly.

The fluctuations of the site energies $\delta \varepsilon_{i}$ are given by the sum of two Gaussian random variables: one with standard deviation $\sigma_{\mathrm{p}}$, completely uncorrelated for different pigments, describing local energy shifts due to the slightly different protein environments; one with standard deviation $\sigma_{0}$, which is the same for all pigments, describing global shifts of the ground-state energy.
Throughout this work, the B800 ring enters the dynamics only during the excitation process, as we describe in more detail in the next section. However, we explicitly included B800 rings in the calculation of the absorption spectra shown in Fig. 2(b) for a better comparison with the experimental data. We model the B800 ring as a set of nine transition dipoles arranged on a circle of radius $3.1 \mathrm{~nm}$, concentric to the $\mathrm{B} 850$ ring and vertically displaced from it by $1.7 \mathrm{~nm}$. We consider the B800 dipoles to be perfectly coplanar and tangent to the circle. In accordance with previous works [51], we set the ratio between B850 and B800 pigment dipole strengths to 1.1. The site energy of B800 pigments $\varepsilon_{\mathrm{B} 800}$ has an average value of $1.2510^{4} \mathrm{~cm}^{-1}$, with Gaussian static disorder with standard deviation $\sigma_{\mathrm{B} 800}=100 \mathrm{~cm}^{-1}$, whereas the single-pigment optical dephasing rate is $\gamma_{\mathrm{B} 800}=70 \mathrm{~cm}^{-1}$ [51].

The B800 pigments couple to each other and to the B850 pigments via dipole-dipole interactions. As a result, one may expect that excitons can, in principle, delocalize across both rings. However, the presence of static disorder is sufficient to destroy any inter-ring delocalization [51]. This case can be seen in Fig. 7, where we plot the average population of an LH2 exciton $|\tilde{\alpha}\rangle$ (diagonalizing the total $\mathrm{B} 800+\mathrm{B} 850$ Hamiltonian) on site $|i\rangle$. The absence of significant B800-B850 coherent mixing thus justifies the approach adopted throughout the paper, where we only consider the indirect effect of incoherent B800-to-B850 energy transfer to populate the B850 manifold after initial laser excitation.

\section{APPENDIX F: SIMULATIONS OF AN LH2 ARRAY}

In this section, we give more details on the simulations of the full LH2 linear array. In analogy with the experiment of Escalante et al. [20], we look at the stationary exciton

TABLE II. Model parameters for a B850 ring.

\begin{tabular}{lcl}
\hline \hline & Value & \multicolumn{1}{c}{ Parameter description } \\
\hline$\theta$ & $10.06^{\circ}$ & Intradimer angle [80] \\
$\Delta \theta_{1}, \Delta \theta_{2}$ & $19.9^{\circ}, 17.6^{\circ}$ & Dipole tilt away from the tangent [80] \\
$\varphi_{1}, \varphi_{2}$ & $6.24^{\circ}, 4.81^{\circ}$ & Dipole tilt away from the ring plane [80] \\
$r$ & $3.0 \mathrm{~nm}$ & Ring radius [64] \\
$\Delta z$ & $0.063 \mathrm{~nm}$ & Vertical displacement between $\alpha$ and $\beta$ Bchl [80] \\
$d$ & $6.4 \mathrm{D}$ & $Q_{y}$ dipole moment of Bchl [81] \\
$\varepsilon$ & $12330 \mathrm{~cm}^{-1}$ & Average site energy [51] \\
$J_{1}, J_{2}$ & $320,255 \mathrm{~cm}^{-1}$ & Intradimer and interdimer nearest-neighbor coupling [82] \\
$\gamma$ & $(20 \mathrm{fs})^{-1}$ & Single-pigment optical dephasing rate [51] \\
$R_{01}$ & $(200 \mathrm{fs})^{-1}$ & Relaxation rate between the two lowest excitons [56] \\
$\Gamma_{\mathrm{th}}$ & $(1 \mathrm{~ns})^{-1}$ & Decay rate from thermalized exciton manifold [39] \\
$T$ & $298 \mathrm{~K}$ & Temperature of the vibrational bath \\
$\phi$ & 0.1 & Quantum yield of fluorescence [39] \\
$\sigma_{\mathrm{p}}, \sigma_{0}$ & Standard deviation of uncorrelated or ground-state disorder [51] \\
$r_{\mathrm{c}}$ & $265 \mathrm{~cm}^{-1}, 33 \mathrm{~cm}^{-1}$ & Correlation radius of local pigment environments [60] \\
\hline \hline
\end{tabular}

${ }^{\mathrm{a}}$ These values have been rescaled from Ref. [51] to match the B850 absorption linewidth. 


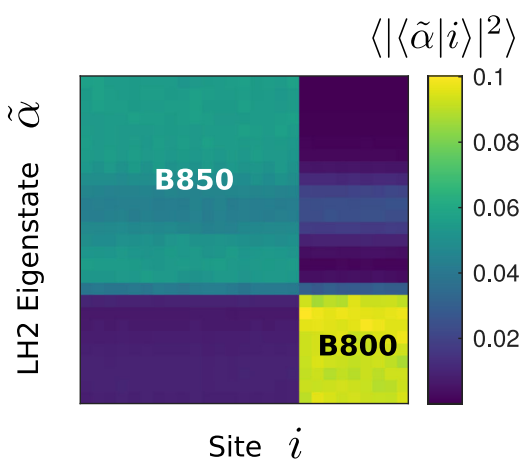

FIG. 7. B800-B850 mixing in a LH2 ring. Disorder-averaged populations of LH2 mixed B800-B850 eigenstates $|\tilde{\alpha}\rangle$ on different sites $|i\rangle$. The eigenstates are clearly divided into two blocks, representing the fact that there is negligible coherent mixing between B800 and B850 excitons. The ensemble average is obtained from $10^{4}$ realizations of static disorder.

probability profile along the linear array upon continuous wave driving; i.e., we numerically look for the steady state of Eq. (3) (with all rates also dependent on the specific subunit $n$ since every subunit exhibits a different realization of static disorder, i.e., different spectra and pigment positions, affecting relaxation, fluorescence, transfer and injection rates). In the experiment, the driving is provided by an $800-n m$ laser with a spatial intensity profile with a full width at half maximum of $400 \pm 50 \mathrm{~nm}$. In order to simplify the numerics, we consider instead injection on a single B850 complex at the center of the chain. In this way, we can simulate shorter chains (up to 1001 subunits) and be safely protected from systematic errors introduced by the finite size of the array. The results for spatially broad excitation can be recovered as shown in Appendix A in the case of the exactly solvable model, i.e., by convolving the result for local injection with the desired excitation profile.

We clarify that our injection profile is local, i.e., $I_{\alpha}^{n} \propto \delta_{n_{0} n}$, where $n_{0}=501$ is the central site of the chain, but we have not specified yet in which states $\alpha$ of the ring excitations are injected. Optical excitation at $800 \mathrm{~nm}$ cannot be absorbed by the B850 subunit [as seen in Fig. 2(b)]; nevertheless, it can excite the B800 ring. We are not explicitly considering the B800 subunit in our model. However, excitations enter the B850 ring upon downhill B800 $\rightarrow$ B850 energy transfer. Because of the small coherent coupling between the two concentric rings, this transfer process is largely controlled by incoherent rates of the form of Eq. (D7); therefore, we can think of an indirect excitation of the B850 excitons with an energy distribution given by

$$
I_{\alpha}^{n_{0}} \propto \frac{\left(\gamma_{\alpha}+\gamma_{\mathrm{B} 800}\right)}{\left(E_{\alpha}-\varepsilon_{\mathrm{B} 800}\right)^{2}+\left(\gamma_{\alpha}+\gamma_{\mathrm{B} 800}\right)^{2}},
$$

where $\varepsilon_{\mathrm{B} 800}$ and $\gamma_{\mathrm{B} 800}$ are the optical gap and dephasing rate of the B800 pigments, whose values are given in
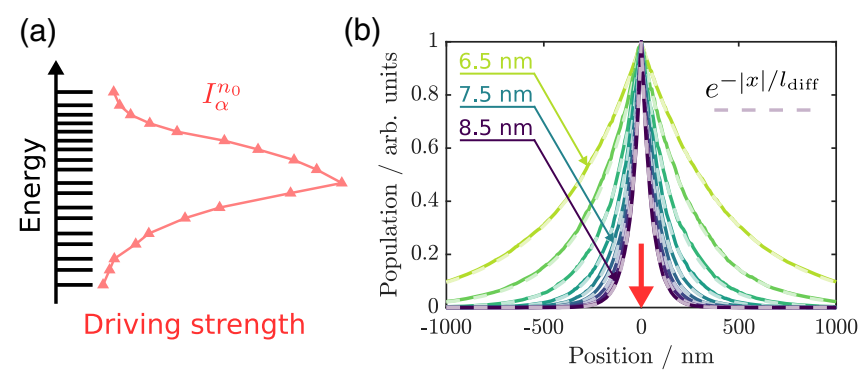

FIG. 8. Driving profile and excitonic stationary spatial distribution. (a) Driving at the central ring takes place in the B800 pigments, with a distribution across the excitonic spectrum given by Eq. (F1). The profile is averaged over $10^{4}$ realizations of static disorder. (b) Injection at a single site (at $x=0$ ) results in an exponential exciton distribution along the light-harvesting array. Results are shown for intercomplex distances ranging from $6.5 \mathrm{~nm}$ to $8.5 \mathrm{~nm}$. The exciton distributions obtained numerically (solid lines) fully agree with exponential distributions of different diffusion length (dashed lines). Numerical results are obtained as averages over $10^{3}$ realizations of static disorder.

Appendix E. The disorder-averaged injection profile (F1) is shown in Fig. 8(a).

The resulting stationary probability profiles are shown in Fig. 8(b) for different lattice steps $l$ ranging from $8.5 \mathrm{~nm}$ to $6.5 \mathrm{~nm}$, after averaging over $10^{3}$ realizations of static disorder. The probabilities $\bar{p}_{\alpha}^{n}$, shown as a function of the position $x=\left(n-n_{0}\right) l$, exhibit a clear exponential decay
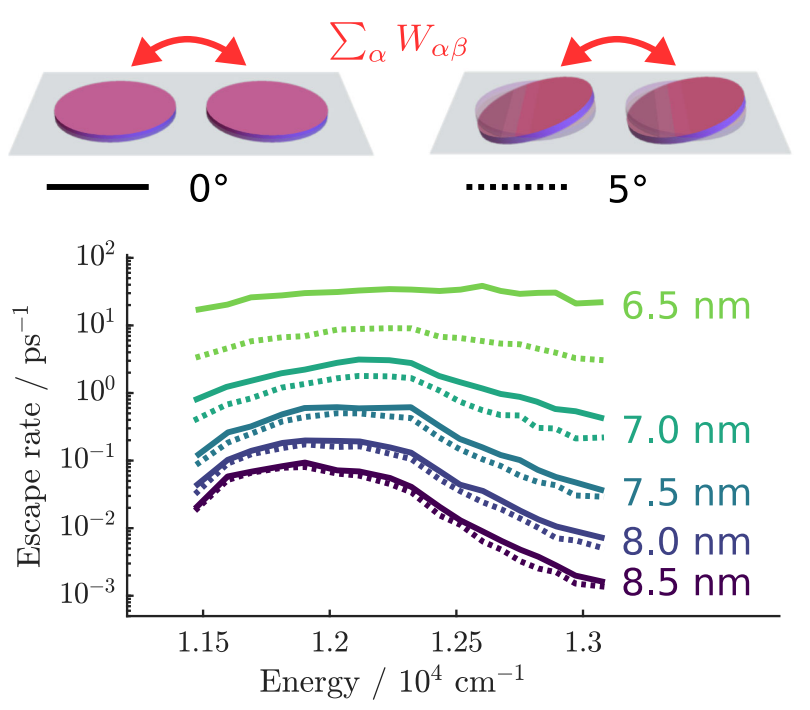

FIG. 9. Energy transfer between tilted B850 rings. Total energy-transfer rate from exciton $\beta$ to any other exciton $\alpha$ on a neighboring ring (escape rate), as a function of the energy of the initial excitonic state $\beta$. Results for coplanar (tilde) rings are shown with solid (dotted) lines, for inter-ring distances ranging from $6.5 \mathrm{~nm}$ (green) to $8.5 \mathrm{~nm}$ (purple). Even a slight tilt of $5^{\circ}$ outside the plane results in a significant reduction of the transfer rate at short inter-ring distances. Results are obtained as averages over $10^{3}$ realizations of static disorder. 
around the injection site in the middle of the array. A convolution of these exciton population profiles with a Gaussian injection profile with 400 -nm full width at half maximum yields the distributions reported in Fig. 3(b).

All simulations are performed for coplanar arrangements of $\mathrm{B} 850$ rings. As discussed in the main text, the nanoengineered arrays analyzed here allow for coplanar B850 rings. However, in the main text, we also note that in biological light-harvesting membranes, an angle of about $5^{\circ}$ relative to the aggregation plane is observed. This small tilt leads to slower energy transfer, especially for short interring distances, as shown in Fig. 9.

Lastly, we note that all simulated LH2 arrays feature a fixed intercomplex distance $l$ throughout the length of the array, which clearly represents an approximation since every realistic macromolecular assembly will show fluctuations in the lattice constant. While these large-scale geometric defects are expected to severely limit the range of ballistic energy transfer and eventually lead to diffusive transport, they only act as an additional source of static disorder in our model, which already describes energy transfer in the diffusive regime. Thus, in our case, this further noise source will lead to changes in the transfer rates but will not affect the scaling of the diffusion length with respect to the lattice constant, leaving our main conclusions unaltered. Nevertheless, the effect of lattice constant fluctuations can be estimated by analyzing the dependence of the diffusion length $l_{\text {diff }}$ on the intercomplex distance $l$, shown in Fig. 3(d), which seems to be described by a monotonically decreasing convex function. In other words, the increase in diffusion length that we obtain by shortening the lattice constant $l$ by an amount $\delta l$ exceeds the decrease that we would obtain by expanding the lattice constant by the same amount to $l+\delta l$. Thus, when assuming a symmetric distribution of lattice constants around $l$, the averaging would lead to a slightly larger diffusion length. However, we note that this effect might be too small to be observed in our case because of the presence of other sources of static disorder.

[1] J. Adolphs and T. Renger, How Proteins Trigger Excitation Energy Transfer in the FMO Complex of Green Sulfur Bacteria, Biophys. J. 91, 2778 (2006).

[2] F. Müh, M. E. Madjet, J. Adolphs, A. Abdurahman, B. Rabenstein, H. Ishikita, E.-W. Knapp, and T. Renger, AlphaHelices Direct Excitation Energy Flow in the Fenna Matthews Olson Protein, Proc. Natl. Acad. Sci. U.S.A. 104, 16862 (2007).

[3] V. I. Novoderezhkin, D. Rutkauskas, and R. van Grondelle, Dynamics of the Emission Spectrum of a Single LH2 Complex: Interplay of Slow and Fast Nuclear Motions, Biophys. J. 90, 2890 (2006).

[4] J. Strümpfer and K. Schulten, Light Harvesting Complex II B850 Excitation Dynamics, J. Chem. Phys. 131, 225101 (2009).
[5] J. Strümpfer and K. Schulten, Excited State Dynamics in Photosynthetic Reaction Center and Light Harvesting Complex 1, J. Chem. Phys. 137, 065101 (2012).

[6] M. Pajusalu, R. Kunz, M. Rätsep, K. Timpmann, J. Köhler, and A. Freiberg, Unified Analysis of Ensemble and SingleComplex Optical Spectral Data from Light-Harvesting Complex-2 Chromoproteins for Gaining Deeper Insight into Bacterial Photosynthesis, Phys. Rev. E 92, 052709 (2015).

[7] X. Hu, T. Ritz, A. Damjanović, F. Authennrieth, and K. Schulten, Photosynthetic Apparatus of Purple Bacteria, Q. Rev. Biophys. 35, 1 (2002).

[8] S. Jang, M. D. Newton, and R. J. Silbey, Multichromophoric Förster Resonance Energy Transfer, Phys. Rev. Lett. 92, 218301 (2004).

[9] F. Caycedo-Soler, F. J. Rodríguez, L. Quiroga, and N. F. Johnson, Light-Harvesting Mechanism of Bacteria Exploits a Critical Interplay between the Dynamics of Transport and Trapping, Phys. Rev. Lett. 104, 158302 (2010).

[10] J. M. Salverda, M. Vengris, B. P. Krueger, G. D. Scholes, A. R. Czarnoleski, V. Novoderezhkin, H. van Amerongen, and R. van Grondelle, Energy Transfer in Light-Harvesting Complexes LHCII and CP29 of Spinach Studied with Three Pulse Echo Peak Shift and Transient Grating, Biophys. J. 84, 450 (2003).

[11] J. Herek, N. Fraser, T. Pullerits, P. Martinsson, T. Polívka, H. Scheer, R. Cogdell, and V. Sundström, B800 $\rightarrow$ B850 Energy Transfer Mechanism in Bacterial LH2 Complexes Investigated by B800 Pigment Exchange, Biophys. J. 78, 2590 (2000).

[12] F. Ma, L.-J. Yu, Z.-Y. Wang-Otomo, and R. van Grondelle, Temperature Dependent LH1 $\rightarrow$ RC Energy Transfer in Purple Bacteria Tch. Tepidum with Shiftable LH1- $Q_{y}$ Band: A Natural System to Investigate Thermally Activated Energy Transfer in Photosynthesis, Biochim. Biophys. Acta 1857, 408 (2016).

[13] R. Agarwal, A. H. Rizvi, B.S. Prall, J. D. Olsen, C. N. Hunter, and G. R. Fleming, Nature of Disorder and InterComplex Energy Transfer in LH2 at Room Temperature: A Three Pulse Photon Echo Peak Shift Study, J. Phys. Chem. A 106, 7573 (2002).

[14] D. I. G. Bennett, P. Malý, C. Kreisbeck, R. van Grondelle, and A. Aspuru-Guzik, Mechanistic Regimes of Vibronic Transport in a Heterodimer and the Design Principle of Incoherent Vibronic Transport in Phycobiliproteins, J. Phys. Chem. Lett. 9, 2665 (2018).

[15] G. D. Scholes, G. R. Fleming, A. Olaya-Castro, and R. van Grondelle, Lessons from Nature about Solar Light Harvesting, Nat. Chem. 3, 763 (2011).

[16] J.-L. Brédas, D. Beljonne, V. Coropceanu, and J. Cornil, Charge-Transfer and Energy-Transfer Processes in $\pi$-Conjugated Oligomers and Polymers: A Molecular Picture, Chem. Rev. 104, 4971 (2004).

[17] M. Baldo and V. Stojanovic, Excitonic Interconnects, Nat. Photonics 3, 558 (2009).

[18] T. Aida, E. W. Meijer, and S. I. Stupp, Functional Supramolecular Polymers, Science 335, 813 (2012).

[19] M. Escalante, P. Maury, C. M. Bruinink, K. van der Werf, J. D. Olsen, J. A. Timney, J. Huskens, C. N. Hunter, V. Subramaniam, and C. Otto, Directed Assembly of Functional 
Light Harvesting Antenna Complexes onto Chemically Patterned Surfaces, Nanotechnology 19, 025101 (2008).

[20] M. Escalante, A. Lenferink, Y. Zhao, N. Tas, J. Huskens, C. N. Hunter, V. Subramaniam, and C. Otto, Long-Range Energy Propagation in Nanometer Arrays of Light Harvesting Antenna Complexes, Nano Lett. 10, 1450 (2010).

[21] I. Eisenberg, S. Yochelis, R. Ben-Harosh, L. David, A. Faust, N. Even-Dar, H. Taha, N. M. Haegel, N. Adir, N. Keren, and Y. Paltiel, Room Temperature Biological Quantum Random Walk in Phycocyanin Nanowires, Phys. Chem. Chem. Phys. 16, 11196 (2014).

[22] C. Vasilev, M. P. Johnson, E. Gonzales, L. Wang, A. V. Ruban, G. Montano, A. J. Cadby, and C. N. Hunter, Reversible Switching between Nonquenched and Quenched States in Nanoscale Linear Arrays of Plant Light-Harvesting Antenna Complexes, Langmuir 30, 8481 (2014).

[23] M. A. Harris, P. S. Parkes-Loach, J. W. Springer, J. Jiang, E. C. Martin, P. Qian, J. Jiao, D. M. Niedzwiedzki, C. Kirmaier, J. D. Olsen, D. F. Bocian, D. Holten, C. N. Hunter, J.S. Lindsey, and P. A. Loach, Integration of Multiple Chromophores with Native Photosynthetic Antennas to Enhance Solar Energy Capture and Delivery, Chem. Sci. 4, 3924 (2013).

[24] E. Boulais, N. P. D. Sawaya, R. Veneziano, A. Andreoni, J. L. Banal, T. Kondo, S. Mandal, S. Lin, G. S. SchlauCohen, N. W. Woodbury, H. Yan, A. Aspuru-Guzik, and M. Bathe, Programmed Coherent Coupling in a Synthetic DNA-Based Excitonic Circuit, Nat. Mater. 17, 159 (2018).

[25] D. M. Eisele, C. W. Cone, E. A. Bloemsma, S. M. Vlaming, C. G. F. van der Kwaak, R. J. Silbey, M. G. Bawendi, J. Knoester, J.P. Rabe, and D. A. Vanden Bout, Utilizing Redox-Chemistry to Elucidate the Nature of Exciton Transitions in Supramolecular Dye Nanotubes, Nat. Chem. 4, 655 (2012).

[26] D. M. Eisele, D. H. Arias, X. Fu, E. A. Bloemsma, C. P. Steiner, R. A. Jensen, P. Rebentrost, H. Eisele, A. Tokmakoff, S. Lloyd, K. A. Nelson, D. Nicastro, J. Knoester, and M. G. Bawendi, Robust Excitons Inhabit Soft Supramolecular Nanotubes, Proc. Natl. Acad. Sci. U.S.A. 111, E3367 (2014).

[27] G. M. Akselrod, P. B. Deotare, N. J. Thompson, J. Lee, W. A. Tisdale, M. A. Baldo, V. M. Menon, and V. Bulovic, Visualization of Exciton Transport in Ordered and Disordered Molecular Solids, Nat. Commun. 5, 3646 (2014).

[28] K. A. Clark, E. L. Krueger, and D. A. V. Bout, Direct Measurement of Energy Migration in Supramolecular Carbocyanine Dye Nanotubes, J. Phys. Chem. Lett. 5, 2274 (2014).

[29] A. T. Haedler, K. Kreger, A. Issac, B. Wittmann, M. Kivala, N. Hammer, J. Köhler, H.-W. Schmidt, and R. Hildner, Long-Range Energy Transport in Single Supramolecular Nanofibres at Room Temperature, Nature (London) 523, 196 (2015).

[30] J. R. Caram, S. Doria, D. M. Eisele, F. S. Freyria, T. S. Sinclair, P. Rebentrost, S. Lloyd, and M. G. Bawendi, Room-Temperature Micron-Scale Exciton Migration in a Stabilized Emissive Molecular Aggregate, Nano Lett. 16, 6808 (2016).

[31] Y. Wan, A. Stradomska, J. Knoester, and L. Huang, Direct Imaging of Exciton Transport in Tubular Porphyrin
Aggregates by Ultrafast Microscopy, J. Am. Chem. Soc. 139, 7287 (2017).

[32] C. Creatore, M. A. Parker, S. Emmott, and A. W. Chin, Efficient Biologically Inspired Photocell Enhanced by Delocalized Quantum States, Phys. Rev. Lett. 111, 253601 (2013).

[33] A. Fruchtman, R. Gómez-Bombarelli, B. W. Lovett, and E. M. Gauger, Photocell Optimization Using Dark State Protection, Phys. Rev. Lett. 117, 203603 (2016).

[34] M. Wertnik, A. Chin, F. Nori, and N. Lambert, Optimizing Co-operative Multi-environment Dynamics in a Dark-StateEnhanced Photosynthetic Heat Engine, J. Chem. Phys. 149, 084112 (2018).

[35] Z. Hu, G. S. Engel, F. H. Alharbi, and S. Kais, Dark States and Delocalization: Competing Effects of Quantum Coherence on the Efficiency of Light Harvesting Systems, J. Chem. Phys. 148, 064304 (2018).

[36] D. M. Rouse, E. M. Gauger, and B. W. Lovett, Optimal Power Generation Using Dark States in Dimers Strongly Coupled to Their Environment, New J. Phys. 21, 063025 (2019).

[37] C. Sissa, A. K. Manna, F. Terenziani, A. Painelli, and S. K. Pati, Beyond the Förster Formulation for Resonance Energy Transfer: The Role of Dark States, Phys. Chem. Chem. Phys. 13, 12734 (2011).

[38] F. Liu, A. V. Rodina, D. R. Yakovlev, A. A. Golovatenko, A. Greilich, E. D. Vakhtin, A. Susha, A. L. Rogach, Y. G. Kusrayev, and M. Bayer, Förster Energy Transfer of Dark Excitons Enhanced by a Magnetic Field in an Ensemble of CdTe Colloidal Nanocrystals, Phys. Rev. B 92, 125403 (2015).

[39] R. Monshouwer, M. Abrahamsson, F. van Mourik, and R. van Grondelle, Superradiance and Exciton Delocalization in Bacterial Photosynthetic Light-Harvesting Systems, J. Phys. Chem. B 101, 7241 (1997).

[40] V. Helenius, R. Monshouwer, and R. van Grondelle, Temperature-Dependent Lifetimes and Quantum Yield of the Singlet and Triplet States of the B820 Subunit of LHI Antenna Complex of Purple Bacterium Rhodospirillum rubrum, J. Phys. Chem. B 101, 10554 (1997).

[41] J. Cao, R. J. Cogdell, D. F. Coker, H.-G. Duan, J. Hauer, U. Kleinekathöfer, T. L. C. Jansen, T. Mančal, R. J. D. Miller, J. P. Ogilvie, V. I. Prokhorenko, T. Renger, H.-S. Tan, R. Tempelaar, M. Thorwart, E. Thyrhaug, S. Westenhoff, and D. Zigmantas, Quantum Biology Revisited, Sci. Adv. 6, eaaz4888 (2020).

[42] S. Lloyd and M. Mohseni, Symmetry-Enhanced Supertransfer of Delocalized Quantum States, New J. Phys. 12, 075020 (2010).

[43] D. F. Abasto, M. Mohseni, S. Lloyd, and P. Zanardi, Exciton Diffusion Length in Complex Quantum Systems: The Effects of Disorder and Environmental Fluctuations on SymmetryEnhanced Supertransfer, Phil. Trans. R. Soc. A 370, 3750 (2012).

[44] G. Yang, N. Wu, T. Chen, K. Sun, and Y. Zhao, Theoretical Examination of Long-Range Energy Propagation in Nanoengineered Light-Harvesting Antenna Arrays, J. Phys. Chem. C 116, 3747 (2012).

[45] T. Pullerits, M. Chachisvilis, and V. Sundström, Exciton Delocalization Length in the B850 Antenna of Rhodobacter sphaeroides, J. Phys. Chem. 100, 10787 (1996). 
[46] M. Chachisvilis, O. Kühn, T. Pullerits, and V. Sundström, Excitons in Photosynthetic Purple Bacteria: Wavelike Motion or Incoherent Hopping?, J. Phys. Chem. B 101, 7275 (1997).

[47] G. Trinkunas, J. L. Herek, T. Polívka, V. Sundström, and T. Pullerits, Exciton Delocalization Probed by Excitation Annihilation in the Light-Harvesting Antenna LH2, Phys. Rev. Lett. 86, 4167 (2001).

[48] Y. C. Cheng and R. J. Silbey, Coherence in the B800 Ring of Purple Bacteria LH2, Phys. Rev. Lett. 96, 028103 (2006).

[49] V. Cherezov, J. Clogston, M. Z. Papiz, and M. Caffrey, Room to Move: Crystallizing Membrane Proteins in Swollen Lipidic Mesophases, J. Mol. Biol. 357, 1605 (2006).

[50] V. May and O. Kühn, Charge and Energy Transfer Dynamics in Molecular Systems (Wiley-VCH Verlag, Weinheim, 2004).

[51] F. Caycedo-Soler, J. Lim, S. Oviedo-Casado, N. F. van Hulst, S. F. Huelga, and M. B. Plenio, Theory of Excitonic Delocalization for Robust Vibronic Dynamics in LH2, J. Phys. Chem. Lett. 9, 3446 (2018).

[52] T. Meier, Y. Zhao, V. Chernyak, and S. Mukamel, Polarons, Localization, and Excitonic Coherence in Superradiance of Biological Antenna Complexes, J. Chem. Phys. 107, 3876 (1997).

[53] S. Davidson, A. Fruchtman, F. A. Pollock, and E. M. Gauger, The Dark Side of Energy Transport along Excitonic Wires: On-Site Energy Barriers Facilitate Efficient, Vibrationally Mediated Transport through Optically Dark Subspaces, J. Chem. Phys. 153, 134701 (2020).

[54] T. Polívka, D. Niedzwiedzki, M. Fuciman, V. Sundström, and H. A. Frank, Role of B800 in Carotenoid-Bacteriochlorophyll Energy and Electron Transfer in LH2 Complexes from the Purple Bacterium Rhodobacter sphaeroides, J. Phys. Chem. B 111, 7422 (2007).

[55] R. Hildner, D. Brinks, J. B. Nieder, R. J. Cogdell, and N. F. van Hulst, Quantum Coherent Energy Transfer over Varying Pathways in Single Light-Harvesting Complexes, Science 340, 1448 (2013).

[56] T. A. C. Stuart, M. Vengris, V. Novoderezhkin, R. J. Cogdell, C. N. Hunter, and R. van Grondelle, Direct Visualization of Exciton Reequilibration in the LHI and LH2 Complexes of Rhodobacter sphaeroides by Multipulse Spectroscopy, Biophys. J. 100, 2226 (2011).

[57] M. H. C. Koolhaas, R. N. Frese, G. J. S. Fowler, T. S. Bibby, S. Georgakopoulou, G. van der Zwan, C. N. Hunter, and R. van Grondelle, Identification of the Upper Exciton Component of the B850 Bacteriochlorophylls of the LH2 Antenna Complex, Using a B800-Free Mutant of Rhodobacter sphaeroides, Biochemistry 37, 4693 (1998).

[58] C. Hofmann, T. J. Aartsma, and J. Köhler, Energetic Disorder and the B850-Exciton States of Individual Light-Harvesting 2 Complexes from Rhodopseudomonas acidophila, Chem. Phys. Lett. 395, 373 (2004).

[59] S. Jang, R. J. Silbey, R. Kunz, C. Hofmann, and J. Köhler, Is There Elliptic Distortion in the Light Harvesting Complex 2 of Purple Bacteria?, J. Phys. Chem. B 115, 12947 (2011).

[60] T. Renger and R. A. Marcus, On the Relation of Protein Dynamics and Exciton Relaxation in Pigment-Protein Complexes: An Estimation of the Spectral Density and a
Theory for the Calculation of Optical Spectra, J. Chem. Phys. 116, 9997 (2002).

[61] T. Pflock, M. Dezi, G. Venturoli, R. J. Cogdell, J. Köhler, and S. Oellerich, Comparison of the fluorescence kinetics of detergent-solubilized and membrane-reconstituted LH2 complexes from Rps. acidophila and Rb. sphaeroides, Photosynth. Res. 95, 291 (2008).

[62] T. J. Pflock, S. Oellerich, J. Southall, R. J. Cogdell, G. M. Ullmann, and J. Köhler, The Electronically Excited States of LH2 Complexes from Rhodopseudomonas acidophila Strain 10050 Studied by Time-Resolved Spectroscopy and Dynamic Monte Carlo Simulations. I. Isolated, Non-interacting LH2 Complexes, J. Phys. Chem. B 115, 8813 (2011).

[63] T. J. Pflock, S. Oellerich, L. Krapf, J. Southall, R. J. Cogdell, G. M. Ullmann, and J. Köhler, The Electronically Excited States of LH2 Complexes from Rhodopseudomonas acidophila Strain 10050 Studied by Time-Resolved Spectroscopy and Dynamic Monte Carlo Simulations. II. Homoarrays of LH2 Complexes Reconstituted into Phospholipid Model Membranes, J. Phys. Chem. B 115, 8821 (2011).

[64] T. Walz, S. J. Jamieson, C. M. Bowers, P. A. Bullough, and C. N. Hunter, Projection Structures of Three Photosynthetic Complexes from Rhodobacter sphaeroides: LH2 at $6 \AA$, LH1 and RC-LH1 at 25 A, J. Mol. Biol. 282, 833 (1998).

[65] S. Jang, S. E. Dempster, and R. J. Silbey, Characterization of the Static Disorder in the B850 Band of LH2, J. Phys. Chem. B 105, 6655 (2001).

[66] L. Cupellini, S. Caprasecca, C. A. Guido, F. Müh, T. Renger, and B. Mennucci, Coupling to Charge Transfer States Is the Key to Modulate the Optical Bands for Efficient Light Harvesting in Purple Bacteria, J. Phys. Chem. Lett. 9, 6892 (2018).

[67] S. J. Jang, Robust and Fragile Quantum Effects in the Transfer Kinetics of Delocalized Excitons between $B 850$ Units of LH2 Complexes, J. Phys. Chem. Lett. 9, 6576 (2018).

[68] S. Scheuring, J. Seguin, S. Marco, D. Lévy, C. Breyton, B. Robert, and J.-L. Rigaud, AFM Characterization of Tilt and Intrinsic Flexibility of Rhodobacter sphaeroides Light Harvesting Complex 2 (LH2), J. Mol. Biol. 325, 569 (2003).

[69] S. Jang, E. Rivera, and D. Montemayor, Molecular Level Design Principle behind Optimal Sizes of Photosynthetic LH2 Complex: Taming Disorder through Cooperation of Hydrogen Bonding and Quantum Delocalization, J. Phys. Chem. Lett. 6, 928 (2015).

[70] S. Scheuring, J. N. Sturgis, V. Prima, A. Bernadac, D. Lévy, and J.-L. Rigaud, Watching the Photosynthetic Apparatus in Native Membranes, Proc. Natl. Acad. Sci. U.S.A. 101, 11293 (2004).

[71] M. B. Plenio and S. F. Huelga, Dephasing-Assisted Transport: Quantum Networks and Biomolecules, New J. Phys. 10, 113019 (2008).

[72] F. Caruso, A. W. Chin, A. Datta, S. F. Huelga, and M. B. Plenio, Highly Efficient Energy Excitation Transfer in Light-Harvesting Complexes: The Fundamental Role of Noise-Assisted Transport, J. Chem. Phys. 131, 105106 (2009).

[73] S. F. Huelga and M. B. Plenio, Vibrations, Quanta and Biology, Contemp. Phys. 54, 181 (2013). 
[74] S. J. Jang and B. Mennucci, Delocalized Excitons in Natural Light-Harvesting Complexes, Rev. Mod. Phys. 90, 035003 (2018).

[75] A. D. Somoza, O. Marty, J. Lim, S. F. Huelga, and M. B. Plenio, Dissipation-Assisted Matrix Product Factorization, Phys. Rev. Lett. 123, 100502 (2019).

[76] Á. Rivas and S. Huelga, Open Quantum Systems: An Introduction, Springer Briefs in Physics (Springer, Berlin, Heidelberg, 2011).

[77] C. M. Marian, Spin-Orbit Coupling and Intersystem Crossing in Molecules, WIREs Comput. Mol. Sci. 2, 187 (2012).

[78] R. Englman and J. Jortner, The Energy Gap Law for Radiationless Transitions in Large Molecules, Mol. Phys. 18, 145 (1970).
[79] T. F. Soules and C. B. Duke, Resonant Energy Transfer between Localized Electronic States in a Crystal, Phys. Rev. B 3, 262 (1971).

[80] F. Caycedo-Soler, C. A. Schroeder, C. Autenrieth, A. Pick, R. Ghosh, S. F. Huelga, and M. B. Plenio, Quantum Redirection of Antenna Absorption to Photosynthetic Reaction Centers, J. Phys. Chem. Lett. 8, 6015 (2017).

[81] T. Pullerits, S. Hess, J. L. Herek, and V. Sundström, Temperature Dependence of Excitation Transfer in LH2 of Rhodobacter sphaeroides, J. Phys. Chem. B 101, 10560 (1997).

[82] G. D. Scholes, I. R. Gould, R. J. Cogdell, and G. R. Fleming, Ab Initio Molecular Orbital Calculations of Electronic Couplings in the LH2 Bacterial Light-Harvesting Complex of Rps. acidophila, J. Phys. Chem. B 103, 2543 (1999). 